\title{
A review of the evolution in the research of antioxidants in olives and olive oil during the last four decades
}

\author{
Apostolos K. Kiritsakis ${ }^{\mathrm{a}^{*}}$, Konstantinos A. Kiritsakis ${ }^{\mathrm{b}}$ and Chrysostomos K. Tsitsipas ${ }^{\mathrm{b}}$ \\ anternational Observatory of Oxidative Stress, in Health and AgriFoods, Thessaloniki, Greece \\ ${ }^{b}$ Department of Food Science \& Technology, Aristotle University, Thessaloniki, Greece \\ ${ }^{*}$ Corresponding author: Apostolos K. Kiritsakis, International Observatory of Oxidative Stress, in Health and AgriFoods, 86 Petrou Syn- \\ dika Street, 54248, Thessaloniki, Greece. Tel: 00302310-836322; Mob. 00306972559099; E-mail: Kyritsak@gmail.com \\ DOI: $10.31665 /$ JFB.2020.11236 \\ Received: July 07, 2020; Revised received \& accepted: August 15, 2020 \\ Abbreviations: EVOO/VOO/ROO, extra virgin/virgin/refined olive oil; HPCOO, high polyphenol content olive oil; OMW/OMWW, olive \\ mill wastes/waste water; COD, chemical oxygen demand; A $\beta$, $\beta$-amyloid peptide; AST/ALT, aspartate/alanine transaminase; HDL/LDL, \\ high/low density lipoprotein; BMI, body mass index \\ Citation: Kiritsakis, A.K., Kiritsakis, K.A., and Tsitsipas, C.K. (2020). A review of the evolution in the research of antioxidants in olives \\ and olive oil during the last four decades. J. Food Bioact. 11: 31-56.
}

\begin{abstract}
Olives and olive oil have established, through centuries, strong bonds with the prosperity and well being of the Mediterranean people. Numerous studies have featured them as main antioxidant sources, based on their phenolic profile, which includes phenolic acids and alcohols, polyphenols, lignans, secoiridoids, oleacein and oleocanthal. Several factors, such as cultivar, fruit maturation, processing methods, storage conditions etc. affect the presence of these significant constituents in the products. Re-use of olive mill waste water has been studied to enrich the phenolic profile of the final product. Olive oil is considered a functional food with extensive use in the food industry. The biological effects of olives and virgin olive oil components include protection against cardiovascular diseases, anti-inflammation action, neuro and endothelial protection etc. Research studies, in vitro and in vivo, in humans and in animals have been performed to better understand the metabolism and bioactivity of olives and olive oil phenolics. Olives and olive oil antioxidants are not only absorbed by the body, but can also attach to the lining of the digestive tract, contributing to the health impact afforded by these products. Laboratory methods and techniques used so far for the qualitative and quantitative identification of these compounds are cited.
\end{abstract}

Keywords: Olives; Olive oil; Olive oil Polyphenols; Antioxidants; Isolation; Determination; Extraction Procedures; Health Impacts.

\section{Introduction}

Olives and olive oil are products extensively consumed in the Mediterranean countries. These unique products apart from being rich in monounsaturated fatty acids, have a unique phenolic profile with distinct physical and health properties (Tresserra-Rimbau and Lamuela-Raventós, 2017). There is also an increasing consumption in extra virgin olive oil (EVOO) worldwide, which is attributed, among others, to its high oxidative stability, due to its chemical composition that includes an unsaponifiable fraction, monounsatu- rated and polyunsaturated fatty acids, and a fraction composed of natural antioxidants such as carotenoids, phytosterols, flavonoids, $\alpha$-tocopherol, and other phenolic compounds (Ramos-Escudero et al., 2015; Romani et al., 2019; Oliveras-Lopez et al., 2014).

Thousands of different polyphenols have been identified in plants, usually in conjugated forms, with one or more sugar residues linked to hydroxyl groups, although linkage with other compounds (amines, carboxylic and organic acids, other polyphenols or lipids) is also possible (Del Rio et al., 2013). According to the number of phenol rings that they contain and the structural ele- 
Table 1. Most important classes of phenolic compounds in olive fruits

\begin{tabular}{|c|c|}
\hline Phenolic group & Compounds \\
\hline Anthocyanins & $\begin{array}{l}\text { Cyanidin-3-glycoside, cyanidin-3-rutinoside, cyanidin-3-caffeyglycoside, cyanidin-3-caffeylrutinoside, } \\
\text { delphinidin 3-rhamosylglycoside-7-xyloside }\end{array}$ \\
\hline Flavonols and flavones & Apigenin-7-glycoside, luteolin-7-rutinoside, luteolin-7-glycoside, luteolin-5-glycoside, quercetin-3-rutinoside \\
\hline Phenolic acids & $\begin{array}{l}\text { Chlorogenic acid, caffeic acid, p-hydroxybenzoic acid, protocatechuic acid, vanillic acid, syringic acid, } \\
\text { p-coumaric acid, o-coumaric acid, ferulic acid, sinapic acid, benzoic acid, cinnamic acid, gallic acid }\end{array}$ \\
\hline Phenolic alcohols & (3,4-Dihydroxyphenyl) ethanol (3,4-DHPEA), ( $p$-hydroxyphenyl) ethanol ( $p$-HPEA) \\
\hline Secoiridoids & Oleuropein, demethyloleuropein, ligstroside, nuzhenide hydroxycinnamic acid derivatives verbascoside \\
\hline
\end{tabular}

Source: Adapted from Ghanbari et al. (2012).

ments that bind these rings, polyphenols are usually divided into several classes, i.e. flavonoids, phenolic acids, stilbenes and lignans. Some polyphenols do not fit in any of these classes and are grouped as "other polyphenols", which is the case of tyrosols in olives and olive oils (Tresserra-Rimbau and Lamuela-Raventós, 2017).

The phenolic compounds in olive oil are simple phenols like phenolic acid and phenolic alcohols (hydroxytyrosol, tysorol), polyphenols (flavonoids, apigenin, luteolin), lignans (pinoresinol, 1-acetoxypinoresinol), secoiridoids (oleuropein, demethyl oleuropein, elenolic acid, ligstroside) and their aglycones, including 3,4-DHPEA-EA or p-DHPEA-EA, oleacein (3,4-DHPEA-EA) and oleocanthal (p-HPEA-EDA) (Kiritsakis et al., 2020). When present in small amounts in food, phenolic compounds are capable of preventing or reducing the oxidation that can lead to a decrease in both nutritional value and sensory quality (Lozano-Sanchez et al., 2011).

Hydroxytyrosol (HT) is one of the strongest natural antioxidants. It is found in olive tree leaves, olive fruit, olive oil, olive pomace and olive mill waste water (OMWW) (Britton et al., 2019; Kiritsakis et al., 2017a). HT and other natural antioxidants can be extracted from olive pomace and other by-products and can be added to olive oil or to other foods as additives to enhance the shelf life (Vitali Čepo et al., 2018). Secoiridoids are responsible for the "pungent" and "bitter" taste of the oil (Keceli et al., 2017). Secoiridoids and lignans are the most concentrated phenolic compounds of olive oil.

This review focuses on characterizing olives and olive oil antioxidants that have a significant effect on human health, based on in vitro and in vivo studies.

\section{Phenolic compounds in olives}

The polyphenol fraction of raw and table olives differs quantitatively and qualitatively from that of Virgin Olive Oil (VOO). Levels are greater in raw olive fruit and table olives than in virgin olive oils since only $2 \%$ of total phenolic compounds move to the oil phase during fruit processing. Furthermore table olive processing methods markedly influence polyphenol levels in the final products (Kailis and Kiritsakis, 2017). Total polyphenol levels in olive fruit are inversely related to water availability. However, vanillin and vanillic acid contents increase in olive fruit of irrigated olive trees compared to those under water stress. Infestation with olive fruit fly (Bactrocera Oleae) also lowers total polyphenol content of olive fruit. Frost-damaged olives have lower levels of secoiridoids (Goulas et al., 2012).

Phenolic compounds are of great importance for color, flavor and nutritional properties of table olives. They are also responsible for the bitter taste and astringency of olive fruit and make olives inedible without processing. The distribution and structure of the chemical constituents of olive fruit depend on parameters such as cultivar, cultivation practices, geographical origin, and maturation stage (Garcia-Serrano et al., 2019; Ghanbari et al., 2012; Kiritsakis et al., 2020). During processing of table olives, analysis of phenolics can determine the necessary degree of debittering treatment and the effects of different processing methods on phenolic content (Crawford et al., 2018). The most important classes of phenolic compounds in olive fruits are given at Table 1 .

Indicative content of hydroxytyrosol is $250-760 \mathrm{mg} / \mathrm{kg}$ for Kalamata olives, $170-510 \mathrm{mg} / \mathrm{kg}$ for Spanish style olives, and 100-340 $\mathrm{mg} / \mathrm{kg}$ for Greek-style naturally black olives (Blekas et al., 2002). The data suggests that overall hydroxytyrosol levels in commonly available table olives range from 100 to $760 \mathrm{mg} / \mathrm{kg}(0.5-3.8 \mathrm{mg} / 5 \mathrm{~g}$ portion) with a theoretical median value of $\approx 300 \mathrm{mg} / \mathrm{kg}(1.5 \mathrm{mg} / 5 \mathrm{~g}$ portion) (Neveu et al., 2010). Exact intake would depend on the bioavailability of hydroxytyrosol from table olives. Overall, turning color olives in brine had the highest polyphenol levels $(\approx 1,200 \mathrm{mg} /$ $\mathrm{kg}$ ), while oxidized olives (Californian-style black ripe olives) had the lowest $(\approx 200 \mathrm{mg} / \mathrm{kg}$ ). In the case of oxidized olives, hydroxytyrosol is lost due to oxidation and polymerization (Charoenprasert and Mitchell 2012). Cultivar differences in polyphenol levels were also observed with Manzanilla $>$ Hojiblanca $>$ Gordal. A 10-fold difference in hydroxytyrosol has been observed between cultivars.

Health promoting qualities of olive polyphenols have been reported extensively (Bulotta et al., 2014). The consumption of $50 \mathrm{~g}$ of table olives provides about $56 \mathrm{mg}$ of polyphenols. High temperature, especially during frying, increases the formation of acrylamide and decreases the amounts of HT, tyrosol, oleuropein and verbascoside. The best way for someone to benefit from table olives is to consume them as they are (Lodolini et al., 2019) or put them in the pot after the heat has been turned off.

The phenolic profile shows drastic changes after olive fruit processing (Mettouchi et al., 2016). Olives cannot be consumed directly after harvest due to their extreme. Bitterness may be removed with alkali $(\mathrm{NaOH})$ treatment, by immersion in a liquid to dilute the bitter compound oleuropein, or by biological processes (Sahin et al, 2013). The alkali treatment hydrolyzes oleuropein into $\beta$-(3,4-dihydroxyphenyl) ethanol, oleoside 11-methylester, and oleoside, making the table olives delicious (Ghanbari et al., 2012). The alkali treatment leads to losses in phenolic contents and antioxidant activity which are found to be cultivar dependent (Mettouchi et al., 2016). The use of partially purified washing water of Spanish-style green olives or preservation liquid of ripe olive as cover brine in the packaging of ripe olives increases the concentration of phenolic compounds in the flesh more than five times (Garcia-Serrano et al, 2019). Among commercial table ol- 
ives, black ripe have the lowest content in phenolic compounds. New emerging technologies such as Ultrasound Assisted Extraction (UAE) could effectively increase the yield of phenolic compounds mainly hydroxytyrosol, oleuropein and rutin from olives and these phenolic compounds could be used as a potential source of natural antioxidants (Deng et al., 2017). High hydrostatic pressure (HHP) is a useful non-thermal technology to be used for the table olives reduction of main microbial groups and to extend the shelf life of the product. Besides, after HHP treatments were performed, the amount of phenolic compounds was similar to fresh table olives (Lodolini et al., 2019).

\subsection{Phenolic analysis of table olives}

Rapid and reliable methods for quantification of hydroxytyrosol, oleuropein and their derivatives are of special interest, since these compounds have been recognized by the EU as agents protecting low density lipoprotein (LDL) against oxidation and thus offering cardiovascular protection. To date, several GC-MS, HPLC-UV or LC-MS methods are available for monitoring phenolic and secoiridoid compounds in olive products (e.g. table olives, olive oil, olive leaves) (Segura-Carretero et al., 2010). The GC-MS methods, as in the case of olive oil, require derivatization and present long analysis time. The HPLC-UV methods have low sensitivity (e.g. $\mathrm{LOQ}=1 \mu \mathrm{g} / \mathrm{mL}$ for hydroxytyrosol) (Zoidou et al., 2010) and are limited by long chromatographic separation times (Segura-Carretero et al., 2010). Additionally, in some cases, HPLC-UV methods are unable to resolve overlapped peaks making the simultaneous quantification of numerous compounds difficult, especially in short separation times. Rapid LC-MS methods have been applied to unprocessed olives but are restricted to the quantification of oleuropein and hydroxytyrosol (Bouaziz et al., 2009). An HPLCOrbitrap MS/MS method for quantification of nine compounds in fresh olives was reported (Kanakis et al., 2013). A rapid ultrahighpressure liquid chromatography (UHPLC) triple, quadruple MS/ MS (QqQ MS/MS) method utilizing dynamic multiple reaction monitoring (dMRM) for the measurement of a range of key bitter and bioactive constituents in olives was developed (Melliou et al., 2015). The method was applied for the simultaneous quantification of hydroxytyrosol, oleuropein, hydroxytyrosol-4-O-glycoside, luteolin-7-O-glycoside, rutin, verbascoside, oleoside-11-methyl ester, 2,6-dimethoxy-p-benzoquinone, phenolic acids (chlorogenic acid, o-coumaric acid), oleuropein and ligstroside aglycons monoaldehydic forms in three different varieties of fresh and processed olives, cured using either dry-salt or California-style black ripe processing methods. This method offers improved sensitivity, selectivity and throughput in comparison with previously reported methods as reviewed by Segura-Carretero (2010).

The HPLC-Orbitrap and the UHPLC QqQ methods present similar LODs and LOQs for oleuropein, hydroxytyrosol, oleuropein aglycon and ligstroside aglycone, the only four common compounds studied using the two methods. The Orbitrap method however, needed a longer analysis time and the quantification range was restricted to higher concentrations (e.g. 1,000-50,000 $\mathrm{ng} / \mathrm{mL}$ as compared to $100-12,500 \mathrm{ng} / \mathrm{mL}$ for oleuropein aglycon and 500-20,000 ng/mL vs 50-6,250 ng/mL for hydroxytyrosol).

\section{Phenolic compounds of olive oil}

The composition of olive oil is $98 \%$ fatty acids, mainly oleic acid (C18:1), and $2 \%$ other minor components, such as squalene, pig-

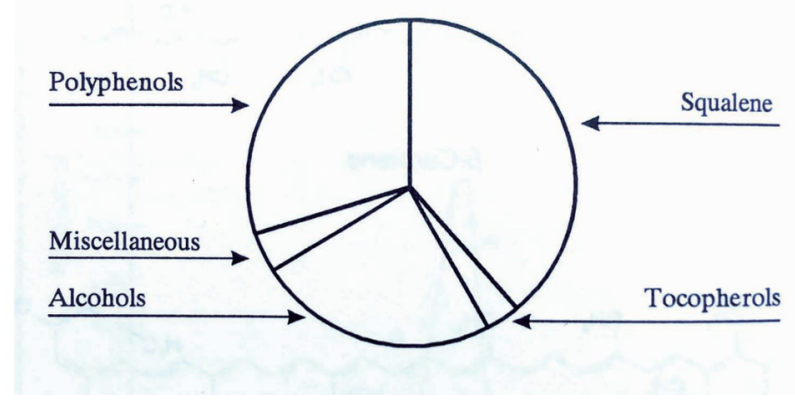

Figure 1. Unsaponifiable fraction of olive oil (Kiritsakis, 1998).

ments, tocopherols, waxes and polyphenols. The polyphenols cover the most significant part of the unsaponifiable fraction of olive oil (Figure 1).

The phenolics of olive oil contain mainly tyrosols, such as hydroxytyrosol, their esterified derivatives with elenolic acid, known as secoiridoids, as well as lignans, flavonoids and phenolic acids.

Oleuropein, a secoiridoid abundant in the Oleaceae family, is an ester of hydroxytyrosol (3,4-DHPEA) and elenolic acid (EA) glycoside, while ligstroside is an ester of tyrosol (p-HPEA) and EA glycoside. Monoaldehydic forms of oleuropein (3,4-DHPEA-EA) and ligstroside (p-HPEA-EA) aglycones are the most abundant secoiridoids in EVOO. Phenethyl alcohols can be linked to dialdehydic form of elenolic acid (EDA), leading to 3,4-DHPEA-EDA and p-HPEA-EDA, known as oleacein and oleocanthal (Celano et al., 2018)

Phenolic acids, which contain at least one phenolic group and a carboxylic acid function (C6-C1 skeleton), represent one third of the total phenolic compounds and could contribute greatly to the biological and sensory properties of EVOO (Tang et al., 2018). The presence of low amounts of benzoic and cinnamic acid derivatives is responsible for the color and organoleptic characteristics, as well as health-related effects (Bendini et al., 2007). Other phenolic acids present in VOO are caffeic, cinnamic, ferulic, $p$ - and o-coumaric, syringic, protocatechuic, and vanillic acids. Hydroxybenzoic acids have a general structure of the C6-C1 type derived directly from benzoic acid. Variations in structure occur due to hydroxylations and methoxylations of the aromatic ring. The overall hydroxybenzoic acids content is generally low in fruits and olive oil. Protocatechuic acid, vanillic acid, gallic acid, syringic acid and p-hydroxybenzoic acid have been found in olive oil in concentrations lower than $3.5 \mathrm{mg} / \mathrm{kg}$ (Montedoro et al., 1992). Gallic, syringic and vanillic acids have been also reported in olive fruits. Vanillic acid was found in olive leaves at a $0.63 \%$ (dry basis) concentration. Phenolic acids with the basic chemical structure of C6-C3 (cinnamic acid) have been found at low concentrations in olive oil $(0.3-1.7 \mathrm{mg} / \mathrm{kg})$, olive fruit and olive leaves, namely caffeic, p-coumaric, o-coumaric, protocatechuic and sinapic acid. The major caffeic acid derivative in olives is verbascoside, a heterosidic ester of caffeic acid, rutinose and dihydroxyphenylethanol. This compound is present in all of the constitutive parts of the olive fruit and, depending on the cultivar, its content can vary between 0.2 and $22 \mathrm{mg} / \mathrm{g}$ of the dry weight of olive fruits. Despite its high content in olives, this compound has not been reported in olive oil.

\subsection{Flavonoids}

Rutin, a flavonol, and luteolin-7-glycoside, a flavone, are the most 
common flavonoids found in olives (Tresserra-Rimbau and Lamuela-Raventós, 2017).The content of flavonoids in olives and olive oil is very low. However, the concentration of these compounds in the fruit during maturation can reach $150 \mathrm{mg}$ per $\mathrm{g}$ of fresh weight. Especially in the last months of fruit maturation, other glycosides including apigenin-7-glycoside, cyanidine-3-glycoside and cyanidine-3-rutinoside can also be found. In olive oil, the flavonoids luteolin $(2-8.4 \mathrm{mg} / \mathrm{kg})$ and apigenin $(0.4-2 \mathrm{mg} / \mathrm{kg})$ have been detected.

\subsection{Phenyl alcohols}

These compounds include molecules containing the $\mathrm{C}_{6}-\mathrm{C}_{2}$ structure corresponding either to 2-(4-hydroxyphenyl) ethanol (p-HPEA, tyrosol, Ty) or to 2-(3,4-dihydroxyphenyl) ethanol (3,4-DHPEA, hydroxytyrosol, HT) (Del Monaco et al., 2015). Hydroxytyrosol and tyrosol derivatives other than glycosides represent the major phenolic fraction of almost all virgin olive oils and olive fruits. Bianchi and Pocci (1994) reported the presence of 3,4-dihydroxyphenylglycol in olives and in vegetation waters. 2-(3,4-dihydroxyphenyl) ethyl acetate (hydroxytyrosol acetate) has also been identified as a component of the phenolic fraction of olive oil (up to $42 \mathrm{mg}$ / $\mathrm{kg}$ ) (Brenes et al., 2002).

Glycosides of hydroxytyrosol were found in olive fruit, olive leaves and in fresh olive oil obtained from cold processing olives (Bianco et al., 2001).

\subsection{Pigments}

The prevalent phenolic compounds in olives and virgin olive oil are the secoiridoid derivatives (Montedoro et al., 1992). Secoiridoids is a sub-class of iridoids, which is a class of regular monoterpenoid lactones containing a cyclopentanodihydropyran ring system, the iridane skeleton.

Secoiridoids are probably formed from carboxylic iridoids by cleavage of the cyclopentane ring followed by functionalization (Bianco et al., 2001). Examples of this class of compounds are secologanin and elenolic acid.

These compounds are not phenolic, however in Olea europaea they are found as esters of tyrosol and hydroxytyrosol. Oleuropein, an heterosidic ester of elenolic acid with hydroxytyrosol is clearly predominant among phenolic compounds in olives. Ligstroside, which is a heterosidic ester of elenolic acid with tyrosol, demethyloleuropein and oleoside, a non phenol, and nüzhenide are also found in high concentration in the fruit (Inouye and Uesato, 1986).

Oleuropein and demethyloleuropein have been found in parts of the fruit such as peel, pulp and seed but they are most frequent in the pulp. In contrast, nüzhenide, a tyrosol derivative, has been found only in the seeds (Gariboldi et al., 1986).

Since the concentration of oleuropein and ligstroside derivatives rise to a great extend during the mechanical extraction of olive oil, nuclear magnetic resonance (NMR) studies on enzymatic hydrolysis of oleuropein by $\beta$-glycosidase have been performed in chloroform/water $(1: 1)$ and in water in order to understand the mechanism of synthesis/biosynthesis of these derivatives (PaivaMartins et al., 2009).

\subsection{Lignans}

Lignans are products of the dimerization of phenylpropene pre- cursors (C6-C3 dimers). The lignans pinoresinol and 1-acetoxypinoresinol have been found in extra virgin olive oil. Pinoresinol concentration ranged from 19 to $65 \mathrm{mg} / \mathrm{kg}$ of olive oil and 1-acetoxypinoresinol concentration ranged from 5 to $97 \mathrm{mg} / \mathrm{kg}$ of olive oil. Lignans seem to be among the most stable phenolics during oil storage and processing (Silva et al., 2018). Lignans are present in the pulp of olive fruit and the woody portion of the seed and are released into the oil during the extraction process without biochemical modification (de Torres et al., 2018). The lignan fraction of VOO characterizes the olive cultivar. 1-acetoxypinoresinol was identified and quantified in olive oil, while lariciresinol and secoisolariciresinol were quantified in olives after hydrolysis. Pinoresinol was quantified in olives and olive oil as well.

\subsection{Hydroxyl-Isochromans}

Hydroxyl-isochromans compounds derive from a reaction between hydroxytyrosol and aromatic aldehydes (benzaldehyde and vanillin). Such a reaction also occurs in a natural matrix, with oleic acid acting as a catalyst. Two compounds of this class, 1-phenyl-6,7dihydroxyisochroman and 1-(3'-methoxy-4'-hydroxy) phenyl-6,7dihydroxy-isochroman, were identified.

The levels of these compounds in samples of extra virgin olive oil are very low and extremely variable, ranging from $8-1,400 \mathrm{ng} /$ $\mathrm{kg}$ for 1-phenyl-6,7-dihydroxy-isochroman and 20-390 $\mathrm{ng} / \mathrm{kg}$ for 1-(3-methoxy-4-hydroxy) phenyl-6,7-dihydroxy-isochroman (Bianco et al., 2001).

The amount of total phenolic content found in EVOO depends on the olive cultivar, agronomic conditions, the methods used to obtain the oil, and storage conditions (Kiritsakis et al., 2020). EVOO may contain between 100 and $800 \mathrm{mg} / \mathrm{kg}$ total polyphenols, while refined oils have a concentration of less than $10 \mathrm{mg} / \mathrm{kg}$ (Neveu et al., 2010). Figure 2 shows the main phenolic substances in olive oil and some representative compounds.

Table 2 shows the concentration of flavonoids, lignans, phenolic acids and other polyphenols in black and green olives and extra virgin, virgin and refined olive oil. Data were obtained from the phenol-explorer database (www.phenol-explorer.eu), the first comprehensive database on polyphenol content in foods, developed by INRA (Institut National de la Recherche Agronomique). Concentrations are derived from the systematic collection of scientific publications and are based on different chromatographic methods. In the case of phenolic acids and lignans from olives, chromatography is applied after hydrolysis, which is needed to release phenolic compounds, otherwise inaccessible for analysis. Black olives have the highest polyphenol content, especially phenolic acids and flavonoids, compared to green olives and olive oils. In contrast, refined olive oils have the lowest values of all polyphenol classes except for lignans.

VOO is considered a functional food, having a high level of monounsaturated fatty acids (MUFAs) and several minor components with biological properties and this fact has included them among the fats with high nutritional quality (Montano et al., 2016). It can be differentiated from all other vegetable oils due to its very particular phenolic composition (Del Monaco et al., 2015; Ghanbari et al., 2012).

EVOO usually contains phenolic compounds that can range between 50 and $1.000 \mathrm{ppm}(\mathrm{mg} / \mathrm{kg})$ depending on the cultivar, pedoclimatic conditions, maturity stage of the fruit and extraction conditions (Kiritsakis, 1998), but values usually range between 100 and $400 \mathrm{mg} / \mathrm{kg}$. According to literature, the monocultivar olive oils from Arbequina, Picual and Cornicabra (Spain), Moraiolo 
Phenolic alcohols<smiles>OCCc1ccc(O)c(O)c1</smiles>

Tyrosol<smiles>OCc1ccc(O)cc1</smiles>

Hydroxytyrosol<smiles>O=C(O)CCC1CCC(O)C1O</smiles><smiles>O=C(O)C=Cc1ccc(O)cc1</smiles><smiles>O=C(O)c1ccc(O)c(O)c1</smiles>

Vanillic acid

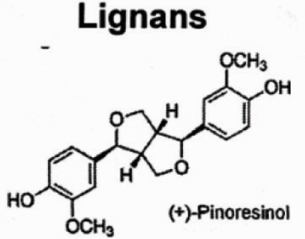

Pinoresinol

Flavonoids

Ferulic acid
Caffeic acid

\section{Secoiridoids}<smiles>CCC(C=O)CC(C=O)CC(=O)OCCc1ccc(O)cc1</smiles>

Oleuropein

Ligstroside

Oleocantal

Figure 2. Main phenolic substances in olive oil.

and Leccino (Italy), Verdeal, Carrasquinha and Cordovil (Portugal), Koroneiki (Greece) and Picholine (Morocco) have usually shown a very high concentration in total phenols. Cultivars such as Arbequina, Manzanilla, Morisca, and Picolimon (from Spain), Queslati, Nebjmel, Swabaâ Algia, and Semni (from Tunisia) and Ayvalik and Nizip (from Turkey) contain lower amounts of phenols (del Monaco et al., 2015).

During olive fruit processing, secoiridoid gluosides such as oleuropein and ligstroside are hydrolyzed by $\beta$-glycosidase, producing more lipophilic secoiridoid derivatives, that are released into the oil during the mechanical extraction process. In contrast, several simple phenols and glycosides, such as oleuropein, being much more hydrophilic, diffuse into the aqueous phase during the oil fruit processing and are mostly lost in the wastewater phase (Kiritsakis and Markakis, 1987).

Lipophilic and hydrophilic phenols are the most important antioxidants in EVOO. Lipophilic phenols in EVOO are tocopherols, which are molecules with a chroman head (with one phenolic and one heterocyclic ring) and a phytyl tail. Different tocopherols vary in the number of methyl substituents and the patterns of substitution in the phenolic ring. Among them, $\alpha$-tocopherol is the most abundant (90\%), but $\beta$ - and $\gamma$-tocopherols are also present (Peres et al., 2016). Another minor compound with great value is the coenzyme Q10 (CoQ10), an endogenous lipophilic compound that is involved in essential cell regulations and modulations, mainly in the mitochondrial respiratory chain. EVOO consumption may be a dietary natural source for increasing intake of CoQ10 ranged from 48 to $85 \mathrm{mg} / \mathrm{L}$ in EVOOs obtained from Spain and Brazil (Borges et al., 2017).

Hydrophilic phenols are compounds that differentiate EVOO from other vegetable oils. The most important are phenolic alcohols (hydroxytyrosol and tyrosol), secoiridoid derivatives, such as the dialdehydic form of elenolic acid linked to tyrosol (p-HPEAEDA) (oleocanthal), the aldehydic form of elenolic acid linked to tyrosol (p-HPEA-EA), 4-(acetoxyethyl)-1,2-dihydroxybenzene, oleuropein aglycone and its methylated form, phenolic acids and derivatives (such as vanillic acid and vanillin, respectively), lignans (pinoresinol and acetoxypinoresinol) and flavonoids such as luteolin and apigenin that affect its sensory, oxidative and antioxidant properties. These compounds originate as a result of the hydrolysis of oleuropein and ligstroside (de Torres et al., 2018). The oleuropein concentration decreases during maturation, while hydroxytyrosol concentration increases. Phenolic compounds present in olive oil are listed in Table 3.

Table 2. Concentration of different polyphenol classes measured in olives and olive oil ( $\mathrm{mg} / \mathrm{kg}$ )

\begin{tabular}{lllll}
\hline \multirow{2}{*}{ Olives and Olive Oil samples } & \multicolumn{3}{c}{ Polyphenol Class } \\
\cline { 2 - 5 } & Flavonoids & Lignans & Phenolic acids & Other polyphenols \\
\hline Black olives, raw & $1,598 \pm 250$ & $00.7 \pm 00.1^{*}$ & $3,075 \pm 199^{*}$ & $2,658 \pm 340$ \\
Green olives, raw & $05.6 \pm 03.1$ & $00.4 \pm 00.1^{*}$ & $2,205 \pm 134^{*}$ & $2,110 \pm 260$ \\
Extra virgin olive oil & $15.3 \pm 05.7$ & $10.8 \pm 01.7$ & $03.1 \pm 00.2$ & $595 \pm 80$ \\
Virgin olive oil & $02.3 \pm 00.2$ & $28.1 \pm 13.6$ & $05.3 \pm 00.4$ & $541 \pm 44$ \\
Refined olive oil & $01.5 \pm 00.6$ & $31.6 \pm 11.7$ & n.d. & $336 \pm 39$ \\
\hline
\end{tabular}

"Refined" oils are deodorized, neutralized and degummed. Concentrations expressed as $\mathrm{mg} / \mathrm{kg}$ (mean \pm standard deviation). *Chromatography after hydrolysis. Data from www. phenol-explorer.eu (accessed Nov. 18 $8^{\text {th }}$ 2019). 
Table 3. Phenolic compounds of olive oil

\begin{tabular}{|c|c|}
\hline Phenolic Group & Compounds \\
\hline Benzoic acid and Derivatives & $\begin{array}{l}\text { 3-Hydroxybenzoic acid; p-Hydroxybenzoic acid; 3,4-Dihydroxybenzoic acid; Gentisic acid; Vanillic } \\
\text { acid; Gallic acid; Siringic acid }\end{array}$ \\
\hline Cinnamic acid and Derivatives & o-Coumaric acid; p-Coumaric acid; Caffeic acid; Ferulic acid; Sinapinic acid \\
\hline $\begin{array}{l}\text { Other Phenolic Acids } \\
\text { and Derivatives }\end{array}$ & $\begin{array}{l}\text { p-Hydroxyphenylacetic acid; 3,4-Dihydroxyphenylacetic acid; 4-Hydroxy-3-methoxyphenylacetic } \\
\text { acid; 3-(3,4-Dihydroxyphenyl)propanoic acid }\end{array}$ \\
\hline Phenyl Ethyl Alcohols & $\begin{array}{l}\text { Tyrosol [(p-hydroxyphenyl)ethanol] or p-HPEA; Hydroxytyrosol [(3,4-dihydroxyphenyl)ethanol] or } \\
\text { 3,4-DHPEA }\end{array}$ \\
\hline Aglycons Secoiridoids & $\begin{array}{l}\text { Oleuropein aglycon or 3,4-DHPEA-EA; Ligstroside aglycon or p-HPEA-EA; Aldehydic form of } \\
\text { oleuropein aglycon; Aldehydic form of ligstroside aglycon }\end{array}$ \\
\hline Dialdehydic Forms of Secoiridoids & $\begin{array}{l}\text { Decarboxymethyl ligstroside aglycon (3,4-DHPEA-EDA); Decarboxymethyl oleuropein aglycon } \\
\text { (p-HPEA-EDA) }\end{array}$ \\
\hline Flavonols & $(+)$-taxifolin \\
\hline Flavons & Apigenin; Luteolin \\
\hline Lignans & (+)-Pinoresinol; (+)-1-Acetoxypinoresinol; (+)-1-Hydroxypinoresinol \\
\hline Hydroxyisochromans & 1-Phenyl-6,7-dihydroxy-isochroman; 1-(3'Methoxy-4'hydroxy)phenyl-6,7-dihydroxy-isochroman \\
\hline
\end{tabular}

Source: Adapted from Romani et al. (2019).

\section{Sensory properties of VOO associated with polyphenols}

Olive oil is generally characterized by its fruitiness, bitterness, and pungency. The characteristic flavor of $\mathrm{VOO}$ is a result of a unique combination of volatile and phenolic compounds. Bitterness and pungency, both positive attributes of olive oil, are related to the quality and amount of polyphenols present. Oleacein, oleuropein aglycone and oleocanthal are the basic cause of bitterness of olive oil (Vitaglione et al., 2015). Olive oil bitterness can be classified as follows:

1. phenolic compounds up to $220 \mathrm{mg} / \mathrm{kg}$ correspond to non-bitter oils or almost imperceptible bitterness

2. phenolic compounds from $220-340 \mathrm{mg} / \mathrm{kg}$ slight bitterness

3. phenolics from 340 to $410 \mathrm{mg} / \mathrm{kg}$ bitter oils

4. phenolics higher than $410 \mathrm{mg} / \mathrm{kg}$ correspond to quite bitter or very bitter oils.

However, pungency (the burning in the back of the throat) is mainly attributed to oleocanthal (Beauchamp et al., 2005). Oleocanthal and oleacein are responsible for the astringency, the perception of puckering in the oral cavity (Sinesio et al., 2015). Furthermore, positive correlations for the aroma descriptors freshly cut grass, leaves, and nuts, and the phenolic compounds were especially observed for oleoside 11-methylester and vanillic acid (Pedan et al., 2019)

The flavor notes, derived from volatile and phenolic compounds, are the main features evaluated in the organoleptic assessment of EVOO. This assessment identifies mainly positive attributes and defects in the oil, and it is critical for the oil's quality classification according to European legislation (Commission Regulation (EEC), n.d No. 2568/91 and further amendments UE Reg. 1348/2013) and the International Olive Council (IOC, 2015) (Genovese et al., 2018). EVOOs' resistance to oxidation depends not only on phenolic profile or fatty acids (FAs), but also on the interaction of these compounds within the same matrix (Montano et al., 2016).

\section{Factors affecting the functional phenolic compounds in olive oil}

The concentration and antioxidant activity of phenolic compounds in VOO is significantly affected by numerous factors such as cultivar, fruit maturity stage (harvesting time) and harvest methods, fruit freshness before extraction, environmental factors (geographical origin, climatic conditions, location, soil, irrigation systems, water stress etc.) extraction process and storage conditions (Alowaiesh et al., 2018; Ben Hasine et al., 2013; Borges et al., 2017; Kiralan et al., 2012; Kiritsakis et al., 2010; Peres et al., 2016; Rodrigues et al., 2019). The ripening stage of olives has a high impact on the oil's yield, quality, stability and sensory characteristics. Irrigation also plays an important role in the productivity of the olive tree, in fruit ripening, and therefore in phenol and volatile composition. Moreover, when early frosts occur, oils extracted from frosted fruit develop sensory defects. In the last years a lot of attention has been given to the main changes on the characteristics of olives and olive oils along fruit ripening, in order to decide the best harvest time (Alowaiesh et al., 2018; Peres et al., 2016).

Recent studies show a strong interest in phenolic transfer during oil milling, malaxation and extraction, in order to increase the phenolic concentration in olive oils (Cecchi et al., 2018). The large amount of wastes and by-products generated during olive oil production process, which traditionally has been considered as a problem, constitute in fact a great source of value-added or functional compounds that have the potential to be used as food additives and/or nutraceuticals (Rosello-Soto et al., 2015). Thus, a more recent approach to exploiting olive-mill wastes has involved the use of processing technologies to fractionate potential high value components from olive processing residues. The most popular valueadded ingredients of OMWW are phenols (e.g. simple phenolic compounds, tannins, flavonols, anthocyanins, etc.), dietary fibers (pectins, oligosaccharides, mannitol) and squalene. Polyphenols as substances with biological activity (antioxidative, antimicrobial etc.) can be used in numerous applications in the pharmaceutical, cosmetics and food industries. Synthetic antioxidants butylated hydroxytoluene (BHT), butylated hydroxyanisole (BHA) and tertiary-butylhydroquinone (TBHQ), etc. have been used substantially as antioxidants in foods, but concerns over their safety have led researchers towards natural antioxidants. These kinds of synthetic antioxidants are substituted by phenolic compounds and 


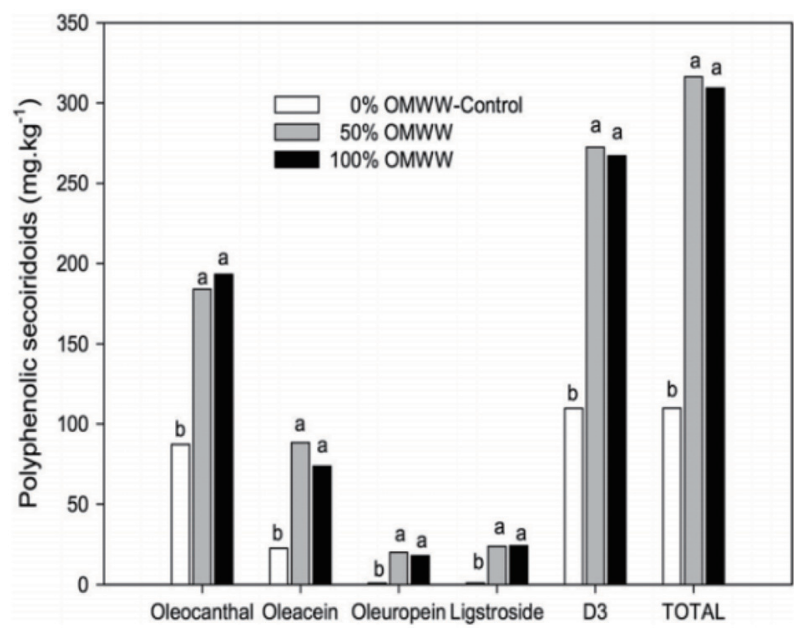

Figure 3. Oleocanthal, oleacein, oleuropein aglycon, ligstroside aglycon, oleocanthal + oleacein (D3) and total phenolic secoiridoids of olive oil enriched by replacing water in a three-phase decanter system with 0 (control), 50 or $100 \%$ OMWW (values within the same letter are not significantly different at 0.05) (Kiritsakis et al., 2017b).

subsequently much of the research on natural antioxidants has also been focused on phenolic compounds, particularly the hydroxycinnamic acids and flavonoids.

The use of olive mill waste water (OMWW) and olive leaf extract (OLE) during olive paste malaxation, gave enriched oils with a higher content in total phenolics, and with higher antioxidant activity. These enriched oils contain higher quantities of oleuropein derivatives and specifically of 3,4-DHPEA-EDA (Figure 3) (Caporaso et al., 2018; Kiritsakis et al., 2017b; 2017c; Venturi et al., 2017).

Many studies on the recovery of polyphenols from OMWW have been conducted on small scale and several techniques are used individually or in combination. These techniques largely comprise membrane separation, extraction, adsorption and chromatographic procedures. Processes of phenols recovery involve typically a condensing step (i.e. thermal concentration, ultrafiltration or lyophilization) prior to carrying out the sequential extraction steps with organic solvents (e.g. methanol, ethanol or hydroalcoholic solutions). Other practices include the application of selective concentration by liquid membranes, resin chromatography or supercritical fluid extraction. These processes aim to either recover a particular phenol (i.e. hydroxytyrosol) in pure form or a phenol mixture as a crude product.

\section{Extraction of phenolics of olive mill waste water (OMWW)}

Solvent extraction is the most widely used technique to recover phenolic compounds from olive mill waste water in spite of its high cost, resulting from the requirement of large amounts of organic solvents. In recent studies, the disadvantages of organic solvents, such as toxicity and flammability, are avoided by using supercritical fluids. Solvent extraction is used individually or preferably employed prior to membrane processes for the recovery of polyphenols from olive oil by-products.

Allouche et al. (2004) achieved high recovery (85.46\%) of hydroxytyrosol from OMWW using a three-stage continuous countercurrent liquid-liquid extraction unit. Emmons and Guttersen (2005) described a process involving addition of citric acid to the raw material, subsequent heating in order to precipitate the solids and extraction of oleuropein aglycon from water immiscible constituent with a non-polar organic solvent mixture, preferably a mixture of 50/50 hexane and acetone. De Martino et al. (2011) proposed a method of obtaining an organic extract containing hydroxytyrosol by means of a Soxhlet, continuous countercurrent or batch extraction system using ethyl acetate, methyl isobutyl ketone, methyl ethyl ketone, diethyl ether, methanol or n-butanol.

Tornberg and Galanakis (2008) disclosed a method for isolating dietary fibers and valuable polyphenols from olive mill wastewater, in which first of all OMWW is defatted by centrifugation and concentrated by removing the water content. Afterwards, it is extracted by using ethanol up to $7 \%(\mathrm{v} / \mathrm{v})$ and an organic acid in the range of $0.5-3 \%$ by weight of the extraction solution. The polyphenols remaining within the dietary fibers were then extracted with at least $85 \%(\mathrm{v} / \mathrm{v})$ ethanol and separated by filtration. After dilution with $15-40 \%(\mathrm{v} / \mathrm{v})$ ethanol, the liquid phase containing polyphenols may be clarified by filtration. Takac and Karakaya (2009) used ethanol up to $70 \%$ and an organic acid in the range of $0.5-3 \%$ to extract polyphenols from OMWW. Lafka et al. (2011) subjected OMWW to conventional liquid solvent extraction and supercritical fluid extraction using different solvents and carbon dioxide, respectively. The optimum extraction conditions were 180 min using a 5:1 (v/w) ratio of ethanol to sample at $\mathrm{pH} 2$. JermanKlen and Mozetic-Vodopivec (2012) used ultrasound-assisted extraction for the recovery of phenols from OMWW. The ultrasoundassisted extraction of freeze-dried OMWW in $100 \%$ methanol $(1.5$ $\mathrm{g} / 25 \mathrm{~mL}, \mathrm{w} / \mathrm{v}$ ) offered high qualitative-quantitative phenol yields and it may be utilized as a valuable source of phenols, especially hydroxytyrosol and tyrosol.

Firlbeck (2013) reported that vanillic acid might be extracted from OMWW quantitatively by a second run of foam fractionation. Foam fractionation of vanillic acid under the optimized process parameters ( $\mathrm{pH}$ of 6-7) led to an enrichment factor of 22.24 in the foamate and $55.88 \%$ recovery in the residue. Yu et al. (2014) tested the possibility of obtaining functional ingredients, such as phenolic compounds, from olive fruit dreg (a waste from olive soft drink processing) using subcritical water extraction. The subcritical water extracts contained higher amounts of phenolic compounds, such as chlorogenic acid, homovanillic acid, gallic acid, hydroxytyrosol, quercetin and syringic acid.

\subsection{Membrane separation}

Olive mill wastes comprise of phenolic compounds of different molecular weights (MW) ranging from low molecular weight phenolics, such as benzoic acid derivatives (MW up to 198), to high molecular weight phenolics, such as secoiridoid aglycons (MW up to 378) and lignans (MW up to 416) (Bendini et al., 2007). The wide range of molecular weights of OMW compounds complicates their recovery with high purities, which may be achieved by using membrane technologies. Selective separation and concentration of phenolic compounds would greatly facilitate their further isolation by other methods. Ultrafiltration in combination with nanofiltration and/or reverse osmosis is usually used for fractionation of OMWW components. The end product was a phenol concentrate that needs further processing for isolation of valuable fractions (Paraskeva et al., 2007). However, when pretreatment is not applied, recovery of membrane permeability with regular cleaning is not possible. Thus, various pretreatment methods have been proposed, such as enzymatic, chemical and physico-chemical pretreatments, neutralization, sedimentation, and centrifugation (Turano et al., 2002). 
Canepa et al. (1988) described a combination of reverse osmosis (RO) and adsorbing on porous polymers for the phenol recovery. Pizzichini and Russo (2005) used microfiltration (MF) and ultrafiltration (UF) for COD reduction, followed by nanofiltration (NF) and RO for phenol recovery. The final RO operation was characterized by spiral-shaped polymeric membranes made of composite polyamide and yielded a concentrate rich in polyphenolic compounds and a permeate consisting of purified water. Castanas et al. (2005) disclosed a system of filters composed of substratums of natural products consisting of turf, sand and sawdust, and optionally, one or more filters of resins consisting of cationic, anionic, mixed type or polyvinylpolypyrrolidone resins. Pizzichini and Russo (2005) proposed a method in which acidified wastewater is subjected to enzymatic hydrolysis by a pectinase enzyme complex to remove the cellulose, hemicellulose and pectin microdispersed components from OMWW. The liquid fraction separated from the degradation products is then used in a membrane system including tangential MF, tangential NF and RO units, in sequence. The ultimate retentate of the RO system is rich in polyphenols. The retentate of MF unit can also be used for extracting polyphenolic compounds thereafter. The experimental study of Russo (2007) concerning removal efficiencies of several MF and UF membranes concentrated the recovered polyphenols by using a final $\mathrm{RO}$ consisting of a polymeric hydronautics membrane (composite polyamide). With a concentration factor of 2.5 , the RO membrane produced a retentate containing $464.870 \mathrm{ppm}$ free low molecular weight polyphenols composed of 390.449 ppm hydroxytyrosol, $27.400 \mathrm{ppm}$ tyrosol, $14.968 \mathrm{ppm}$ oleuropein, $14.329 \mathrm{ppm}$ caffeic acid and $17.724 \mathrm{ppm}$ protocatechuic acid.

Paraskeva et al. (2007) compared the concentration efficiencies of NF and RO after a UF process. The NF step showed reduction of efficiencies to $99 \%$ for TSS, $97 \%$ for TOC, $97 \%$ for COD and $98 \%$ for phenols; the NF retentate had a concentration of $9.96 \mathrm{~g} / \mathrm{L}$ of phenols. Operating the UF permeate with the RO membrane obtained similar reduction results, whereas the phenols concentration in the RO concentrate was $6.78 \mathrm{~g} / \mathrm{L}$. Nunes et al. (2007) described the production of a hydroxytyrosol concentrate by use of nanofiltration and reverse osmosis procedures. An aqueous extract (olive cake plus water) was given to a NF membrane Desal DK type yielding a recovery of hydroxytyrosol of about $70 \%$. The following RO process further concentrated the obtained NF retentate using a Dow Filmtec SW 30 membrane. Villanova (2008) described one NF and one RO module for the concentration of pretreated OMWW. Placed in the third unit after two UF modules, the NF membrane and following RO membrane yielded more than $1 \mathrm{~g} / \mathrm{L}$ of hydroxytyrosol and more than $0.6 \mathrm{~g} / \mathrm{L}$ of tyrosol.

El-Abbassi et al. (2012) investigated the use of direct contact membrane distillation (DCMD) for the concentration of phenolic compounds employing a Gelman TF200 membrane made of PTFE. The obtained retentate had a polyphenols content of about $6.88 \mathrm{~g} / \mathrm{L}$. Garcia-Castello et al. (2010) investigated the concentration abilities of osmotic distillation (OD) and vacuum membrane distillation (VMD) after a NF process. The whole membrane system delivered a liquid concentrate containing about $0.5 \mathrm{~g} / \mathrm{L}$ low MW polyphenols. Servili et al. (2011) described a RO process using a spiral thin-film membrane made of Durasan ${ }^{\mathrm{TM}}$ and polysulfone for the concentration after pretreatments with MF and UF. The content of total phenols rose from 4.9 to $19.3 \mathrm{~g} / \mathrm{L}$ in the $\mathrm{RO}$ concentrate. Cassano et al. (2013) investigated a process based on two UF processes followed by a NF treatment. This permitted a fractionation of OMWW separating organic substances of different molecular weights. The NF retentate was a concentrated solution enriched in polyphenolic compounds suitable for cosmetic, food and pharmaceutical industries as liquid, frozen, dried or lyophi- lized formulations.

El-Abbassi et al (2014) used membrane filtration as a pretreatment prior to application of cloud point extraction to OMWW. The method resulted in a mixture of natural antioxidants suitable for pharmaceutical, cosmetic or food applications. Zagklis et al. (2015) used reverse osmosis, after a nanofiltration, to concentrate the low-molecular-weight compounds, prior to further treatment with resin adsorption/desorption for the purification of the phenolic compounds of OMWW. Comandini et al. (2015) treated OMWW with a semi-industrial membrane filtration system, including ultrafiltration and reverse osmosis modules. Ultrafiltration reduced phenol concentration by about $40 \%$ with respect to the initial level; in the permeate of reverse osmosis, the phenol concentration ranged from 0 to $1 \%$ of the initial content. In contrast, the content of phenolic compounds was increased about 2.6 fold in reverse osmosis concentrate. 3,4-(dihydroxyphenyl) ethanol and p-(hydroxyphenyl) ethanol were the main compounds detected of a total of thirty two.

During filtration of wastewater, severe fouling of the membrane occurs, thus affecting the process performance. Fouling reduces the permeate fluxes and determines both efficiency decrease and variation of membrane selectivity; it also makes the process highly expensive due to repeated plant shut-down for cleaning and washing the membranes (Turano et al., 2002). The critical flux method seems to be one of the most used approaches to overcome fouling problems. At the critical flux point, the drag forces on the solute molecules concentrated over the membrane surface are equal to the dispersive forces, leading to a steady-state layer in a gel state. Under these conditions, only reversible fouling can occur, which can be periodically soft-cleaned. However, some authors have pointed out that operation below the critical flux may not be sufficient to avoid long-term fouling (Stoller and Chianese, 2006). These authors introduced the concept of sustainable flux, at which the desired separation can be operated in a profitable manner, only minimizing but not eliminating fouling entirely. Thus, the best permeate flux cannot be theoretically predicted, but only experimentally measured. Ochando-Pulido et al. (2015) achieved successfully complete restoration of the membrane permeability by performing a cleaning procedure upon turbulent tangential velocity over the membrane $\left(4.01 \mathrm{~m} / \mathrm{s}\right.$, equivalent to $\left.N_{\text {Reynolds }}=21,000\right)$ at a cleaning operating temperature ranging from 30 to $35^{\circ} \mathrm{C}$ for 20-25 min. Dammak et al. (2015) studied a model of oleuropein solution filtration using NF membrane and different feed concentrations and showed that the permeate flux was governed mainly by the osmotic pressure of oleuropein. The flux declined following the accumulation of oleuropein at the membrane surface, forming a concentration polarization layer with lower hydraulic resistance than membrane resistance.

\subsection{Adsorption}

The profitability of an industrial process for the adsorptive purification and concentration of phenolic compounds from OMWW mainly depends on the adsorption efficiency and on the recovery rates during desorption. So far, few studies have been carried out using sorbent materials for the removal of polyphenolic compounds contained in OMWW.

Al-Malah et al. (2000) used activated clay and reported that sorption of phenols was reversible and mainly due to hydrophobic interactions. Cuomo and Rabovskiy (2001) described a method of extracting antioxidant components from olive by-products, which includes the steps of passing OMWW through a solid matrix to trap antioxidants on the matrix and washing the matrix with a po- 
lar organic solvent to remove the antioxidants in the polar organic solvent. Suitable solvents include polar alcohols, acetone, ethyl acetate, acetonitrile, dioxane and mixtures thereof. The polar organic solvent can be partially removed to form a liquid concentrate or preferably substantially and completely removed to produce a solid antioxidant composition. The extracted antioxidant composition has a total phenolic content of about $10-30 \%$ gallic acid equivalents by weight on a dry weight basis and consists of about $1-5 \%$ hydroxytyrosol, $0.4-1.5 \%$ tyrosol and $0.05-1 \%$ oleuropein, all based on dry weight. A particularly preferred solid matrix material is a polymeric adsorbent marketed under the trademark Amberlite ${ }^{\circledR}$.

Activated carbon obtained from treated olive pomace has been used for OMWW polyphenol sorption, succeeding efficient phenol removal. Wilhelm et al. (2003) described the isolation of antioxidants from OMWW by fluidized bed adsorption, especially using polymeric ion-exchanging adsorbents (e.g. Amberlite XAD or Lewatit EP) followed by elution of the adsorbed antioxidants and removal of the solvent. Sabbah et al. (2004) proposed a method using sand filtration and subsequent treatment with powdered activated carbon, yielding a 95\% removal of phenolic compounds. The recovery yield was $60 \%$ using a solid phase extraction by employing Amberlite XAD16 resin as the adsorbent and ethanol as the biocompatible desorbing phase (Scoma et al., 2011). Santi et al. (2008) treated OMWW with mineral adsorbents and concluded that zeolite, compared to other substrates (clay soil and bentonite), appeared to be a useful mineral in reducing the organic load from the OMWW. Bertin et al. (2011) suggested that Amberlite XAD7, XAD16, IRA96 and Isolute ENV+ are the four most promising adsorption resins. The highest recovery of hydroxytyrosol (77\%) was achieved when non-acidified ethanol was used as the desorbing phase.

Ferri et al. (2011) reported the highest phenol adsorption (76\%) using IRA96 polar resin, whereas Ena et al. (2012) stated that granular activated carbon can be more efficient than Azolla in terms of phenols adsorption and desorption. Zagklis et al. (2015) used non-ionic XAD4, XAD16 and XAD7HP resins for the recovery of phenols from low-molecular-weight compounds of OMWW and for their separation from carbohydrates. The recovered phenolic compounds were concentrated through vacuum evaporation reaching a final concentration of $378 \mathrm{~g} / \mathrm{L}$ in gallic acid equivalents containing $84.8 \mathrm{~g} / \mathrm{L}$ hydroxytyrosol. Frascari et al. (2016) developed a continuous-flow adsorption/desorption process for the recovery of phenolic compounds from OMWW (pretreated with centrifugation and microfiltration to remove suspended solids) using an Amberlite XAD16 resin packed in a $0.53 \mathrm{~m}$ column and acidified ethanol as desorption solvent.

Stasinakis et al. (2008) investigated total phenol removal efficiency from OMWW by several types of treated olive pomace (dried olive pomace, dried and solvent extracted olive pomace and dried, solvent extracted and incompletely combusted olive pomace). Singh et al. (2008) investigated the adsorption of both phenol and 2,4-dichlorophenol through the acid treatment of coconut shells, whereas Achak et al. (2009) used banana peel as a lowcost solution biosorbent for removing phenolic compounds from OMWW yielding a $88 \%$ removal with a peel dosage of $30 \mathrm{~g} / \mathrm{L}$.

\subsection{Chromatographic separation}

Fernandez-Bolanos et al. (2002) suggested a method for obtaining hydroxytyrosol from OMWW by a two-step chromatographic treatment. In the first column, the polystyrene based non-activated ion exchange resins, in the form of a gel or macro-reticular, is used to provide partial purification of hydroxytyrosol. After elution with water, the containing hydroxytyrosol solution from the previous stage is fed to the second column involving nonionic, polystyrene based XAD resins to enable hydroxytyrosol to be completely purified after eluting with 30-33\% methanol-water or ethanol-water mixture. Liu and Wang (2008) developed a method for the recovery of hydroxytyrosol involving dilution with water, passage through a styrene resin chromatographic column, washing with distilled water, extraction with ethyl acetate and finally distillation at low temperatures.

\section{Qualitative and quantitative analysis of olive oil phenolic compounds}

Analysis of phenols from olive oil is usually carried out by partitioning the analytes between two phases: both liquid-liquid extraction (LLE) and liquid-solid or solid-phase extraction (SPE). Recently, microdialysis has also been used for polyphenol extraction from EVOO (Bazzu et al., 2017). According to literature, the most common analytical techniques used for the separation and determination of phenolic compounds in olive oil are, so far, highperformance liquid chromatography (HPLC) coupled with ultraviolet or diode array detection (PDA), electrochemical detection, mass spectrometry (MS), gas chromatography coupled to MS, capillary electrophoresis (CE) with UV or MS, Infrared Spectroscopy in the near (NIR) and mid (MIR) regions, Nuclear Magnetic Resonance (NMR) Spectroscopy, and Fourier Transform Infrared (FTIR) Spectroscopy. Recently, Liquid chromatography-electrospray ionization-quadrupole time-of-flight mass spectrometry (LC-ESI-QTOF MS) and HPLC-PDA/ESI-MS methods have been developed and validated for the analysis of the most representative phenolic compounds in EVOO samples (Fanalli et al., 2018; Monasterio et al., 2017). Regardless of the approach selected, the obtained data are subsequently treated using appropriate chemometric tools, including unsupervised methods (mainly PCA and Hierarchical Cluster Analysis (HCA)), and supervised ones (such as Linear Discriminant Analysis (LDA), PLS-DA, SIMCA, k-NN, etc.) (Bajoub et al., 2017).

\subsection{Extraction methods of phenolic compounds from olive oil}

The extraction of phenolic compounds from a sample of olive oil is a very important step for subsequent analyses. The oil extracts usually contain a complex and heterogeneous mixture of phenolic constituents, which makes the selection of the appropriate extraction process a critical step. The aim of the extraction procedure is to obtain the desired polar phenolic fraction from the total mixture with the highest possible efficiency, and to avoid possible structural changes that can occur in the compounds initially present in the extract due to hydrolysis, oxidation or isomerization.

Many methods have been proposed for the extraction of the polar phenolic constituents of the oil, most of which are based on liquid - liquid extraction (LLE) and solid phase extraction (SPE). These methods have been used with many variations by different researchers, who use a variety of extraction solvent systems depending on the objective of their studies.

\subsubsection{Liquid-liquid extraction (LLE)}

This method has commonly been used for the extraction of the 
phenolic fraction of the oil. In a typical protocol, a quantity of oil is dissolved in a lipophilic solvent, usually hexane, and the phenolic fraction is extracted with methanol or a methanol/water mixture (water concentration from 0 to $40 \%$ ). Extraction is carried out either by simple stirring or by sonication for a few minutes. The lipophilic and the methanolic/aqueous phase are separated by centrifugation. The two phases are separated and the methanolic/aqueous phase is evaporated using a rotary evaporator. The extraction with hexane is repeated to achieve better degreasing and the extract is obtained again after evaporation. The use of a suitable solvent system is a first step that greatly affects the performance of the method, as well as, the nature of the solvent selected and the relative proportions of the solvents determine the recovery of polyphenols in the final extract. In addition, other solvents such as petroleum ether and chloroform have been suggested. However, the addition of hexane or other organic solvents in the oil prior to extraction, does not lead to a significant improvement in the recovery of the polyphenolic components (Tasioula-Margari and Okogeri, 2001).

The use of ultrasound, in comparison with the simple stirring, has been studied by Klen and Vodopivec (2012). The results showed that although the simple stirring gave satisfactory results, the use of ultrasound during the extraction led to optimum recovery of the phenolic fraction. In the same contribution, a variant of this method by cooling the sample to $-25^{\circ} \mathrm{C}$ for $2-3 \mathrm{~h}$ was presented. Using this modification a better removal of the non-polar fraction was achieved. Furthermore, Montedoro et al.(1992) had reported several extraction methods for non-hydrolyzable and hydrolyzable phenolic compounds in virgin olive oil, using different solvents at different solvent to test material ratio and other conditions. It was concluded that the optimal solvent system was methanol/water $(80: 20 \mathrm{v} / \mathrm{v})$. Angerosa et al. (2000) later showed opposite results. The incomplete recovery of some components and the formation of emulsion between water and methanol resulted in selection of pure methanol for extraction.

Cortesi et al. (1995) tried to extract the polar components of the oil using tetrahydrofuran (THF)/water $(80: 20 \mathrm{v} / \mathrm{v})$ followed by centrifugation; thus the recovery was five times greater for hydroxytyrosol and twice for tyrosol relative to the methanol/water system $(60: 40 \mathrm{v} / \mathrm{v})$. The use of N, N-dimethyl formamide was shown to have interesting results regarding the recovery of phenolic components. After the process of liquid-liquid extraction and in order to isolate the desired analytes from interfering species, the residual oil was removed by storing at ambient temperature overnight and further centrifugation or extraction with hexane.

Karkoula et al. (2014) used cyclohexane as a lipophilic solvent and acetonitrile for the recovery of the phenolic fraction. In this protocol, $5 \mathrm{~g}$ of oil were dissolved in $20 \mathrm{ml}$ cyclohexane and the solution was agitated for $1 \mathrm{~min}$. Twenty five $\mathrm{mL}$ of acetonitrile were then added and the mixture was agitated for $1 \mathrm{~min}$. The separation of the two phases was achieved by centrifugation for $5 \mathrm{~min}$.

\subsubsection{Solid phase extraction (SPE)}

The great flexibility of the SPE led researchers to use of this technique to recover phenolic compounds from olive oil. The widespread use of this technique also provided opportunity for use of many solvent systems, both for isolation and for purification of the extract. The solid phase extraction includes two modes, the normal phase and the reversed phase. Different sorbents have been used, with C18-cartridges being the most common. Ion exchange cartridges have also been used for the isolation of phenolic fractions from various oils, but the recoveries obtained were quite low (53-62\%). Amino-phase and diol-bonded cartridges, which are in the middle between silica normal and reverse phase, and also compatible with a wide range of solvents have been used (Rios et al., 2005). C-18 is a reliable material for the isolation of simple phenolic substances. However, the recovery is low in the case of secoiridoid derivatives and mainly of their dialdehyde forms. Liberatore et al. (2001) compared different sorbents of SPE with LLE.

\subsubsection{Liquid-liquid microextraction}

In an effort to compare the methods based on liquid-liquid extraction (LLE), liquid-liquid microextraction (LLME) and solid phase extraction (SPE), Pizarro et al. (2012) compared these methods with ultrasonication extraction (USE). The results showed that the application of USE had the highest rate of recovery, however, they observed that the differences of the respective results of the LLME process were not significant. Taking into account that the microextraction has less solvent consumption and needs less time, this method seems to be most suitable for the extraction of phenolic fractions of olive oil.

\section{Analytical approaches to characterize the phenolic profile of olives and olive oils}

Several qualitative and quantitative methods for the analysis of the phenolic content of olive oil and table olives are described by Melliou et al. (2015). The extraction of phenolic compounds from a sample of olive oil is a very important step for subsequent analyses. The aim of the extraction procedure is to obtain the desired polar phenolic fraction from the total mixture with the highest possible efficiency, and to avoid possible structural changes that can occur in the compounds initially present in the extract due to hydrolysis, oxidation or isomerization.

A number of methods proposed for the extraction of the polar phenolic constituents of the oil are based on liquid - liquid extraction (LLE), solid phase extraction (SPE) and Liquid-Liquid Microextraction (LLM). These methods have been used with many variations by different researchers, who use a variety of extraction solvent systems depending on the objective of their studies. The qualitative and/or quantitative determination of the phenolic constituents of the polar extract are mainly based on spectrophotometry or chromatography.

\subsection{Photometric measurement}

A most popular method that is commonly used for quantitation of total phenolics in olive oil is based on the Folin-Ciocalteu reagent that reacts with the hydroxyl groups of the phenolic compounds (Gutfinger et al., 1981). The method includes calibration using a pure standard (e.g. caffeic acid or gallic acid), extraction of phenols from the sample, and measuring the absorbance at $500-750 \mathrm{~nm}$ after the colorimetric reaction. The widespread use of this method is attributed to its simplicity, ease and speed of analysis. However, the drawback of this colorimetric method is its low selectivity, as the color reaction is performed with any phenolic hydroxyl group of the oil components or other oxidizable functional groups.

\subsection{Chromatographic analysis}

Chromatography is the most common method for qualitative and 
quantitative determination of the phenolic content of olive oil. Thin layer chromatography, gas chromatography and liquid chromatography have been used with several types of detectors. HPLC-UV remains the most frequently used method despite the problems that make it inappropriate for quantitative measurement of specific compounds (e.g. oleocanthal). Significant improvements have been achieved using liquid chromatography-mass spectrometry (LC-MS) but still there are ingredients that cannot be measured chromatographically.

\subsection{Thin layer chromatography}

The older chromatographic techniques that were used for the analysis of olive oil phenolics included TLC as well as paper chromatography. Today these techniques are used mainly for final purification of phenolics (Karkoula et al., 2014).

\subsection{Gas chromatography (GC) and GC-MS}

The qualitative and quantitative determination of several phenolic constituents of the oil can be achieved using GC. Although the method allowed the characterization of simple components, other phenols present in large quantities could not be detected. Improvements were made using more sophisticated analytical techniques such as GC-MS and GC-MS/MS which are mainly applied to silylated derivatives of the phenolic components (Saitta et al., 2002). Although there are several analytical and applied works using this technique, other chromatographic techniques like HPLC are more commonly used because they avoid the derivatization step and the high temperatures that can decompose some of the analytes.

\subsection{High pressure liquid chromatography (HPLC)}

The low volatility of many phenolic constituents of olive oil has limited the use of GC method for their qualitative and quantitative determination, and led to the use of the HPLC method as the more popular technique. Numerous mobile phases have been used, but the binary systems consisting of an aqueous component and a less polar organic component such as acetonitrile or methanol remain the most common. Acids (acetic, formic, phosphoric) are commonly added to the two components to maintain a constant acid concentration during fractionation. The routine detection by HPLC is based on the UV absorption at 240, 250 or $280 \mathrm{~nm}$ (Tsimidou et al., 1992).

\subsection{Liquid chromatography (LC)-MS}

The combination of liquid chromatography with mass spectrometry has made a significant change in the analysis of polar components of the oil, as evidenced by the increased number of publications that use this technique during the last years (SeguraCarretero et al., 2010). Electrospray ionization (ESI) in negative mode is more sensitive for the majority of phenolic ingredients and has also been extensively used (Jerman-Klen et al., 2015).

Using LC-MS/MS a serious overlapping problem observed in HPLC-UV methods has been overcome, but the method still suffers from lack of commercial standards and possible formation of artifacts. To avoid problems related to the reaction of some constituents (e.g. oleocanthal or oleacein) with the chromatographic solvents, an interesting approach using derivatization reactions combined with ultra (U) HPLC-ESI-MS/MS has been reported (Di Donna et al., 2011).

\subsection{Capillary electrophoresis (CE)}

Capillary electrophoresis combines reduced analysis time and satisfactory separation of the phenolic fraction. CE is an alternative or additional method of HPLC. The speed, resolution, and ease of use combined with the low cost of the device, makes this technique a useful option for the development and improvement of methods of analysis of olive oil. Vulcano et al. (2015) used CE for quantitation of oleocanthal and oleacein.

\subsection{Quantification using 1D qNMR}

Melliou et al. (2015) reported that to overcome quantification problems, it is necessary to develop a method for olive oil extraction and analysis without involving any reacting mobile or stationary phase. Quantitative nuclear magnetic resonance (qNMR) appeared as an attractive alternative solution to this complicated problem. NMR spectroscopy is well suited for quantitative analyses of complex chemical mixtures. Among the available qNMR methods, 1D ${ }^{1} \mathrm{H}$ NMR typically provides the highest sensitivity with excellent linear response to component concentrations.

\subsection{NMR Spectral analysis of oleocanthal and oleacein in EVOO}

The method is based on the observation that the ${ }^{1} \mathrm{H}$ NMR spectrum of olive oil acetonitrile extracts when recorded in $\mathrm{CDCl}_{3}$ and in magnetic fields of 600 or $800 \mathrm{MHz}$ presented a very well resolved set of peaks corresponding to the aldehydic protons of the target compounds between 9.1 and $9.8 \mathrm{ppm}$ (Figure 4). This spectrum region in all the studied samples was clearly resolved making the integration of the corresponding peaks and their comparison with the peak of the internal standard feasible. Oleocanthal (4), oleacein (3), oleuropein aglycone (5a) and ligstroside aglycone (6a) were quantified by integrating the peaks noted in Figure 4 and by using calibration curves constructed with pure compounds added to oils of zero phenolic content as described by Karkoula et al. (2014). The peaks corresponding to compounds $7 \mathrm{a}, 7 \mathrm{~b}, 9$ and $8 \mathrm{a}, 8 \mathrm{~b}, 10$ were integrated as two total sets corresponding to oleuropein aglycone dialdehyde and enolic forms and to ligstroside aglycon dialdehyde and enolic forms, respectively.

\subsection{Colorimetric quantitation of oleocanthal and oleacein}

A new colorimetric method for the measurement of oleocanthal and oleacein in olive oil has been reported (Magiatis and Melliou, 2015). The measuring method is based on the selective reaction of the polar aldehydes found in olive oil towards the formation of green colored water-soluble derivatives (Schiff bases) that can be quantified in the aqueous phase either by the intensity of the color or by measurement of the absorbance in the visible spectrum. The reaction is achieved under acidic conditions using aniline derivatives bearing polar groups (e.g. carboxyls). As an example of appropriate reagent p-hydroxyanthranilic acid is reported in combination with glacial acetic acid. This method is highly selective and can accurately quantify the oleocanthal and oleacein levels in olive oil. In a large number of olive oils (especially of Greek origin), oleocanthal and oleacein were the dominating tyrosol and 


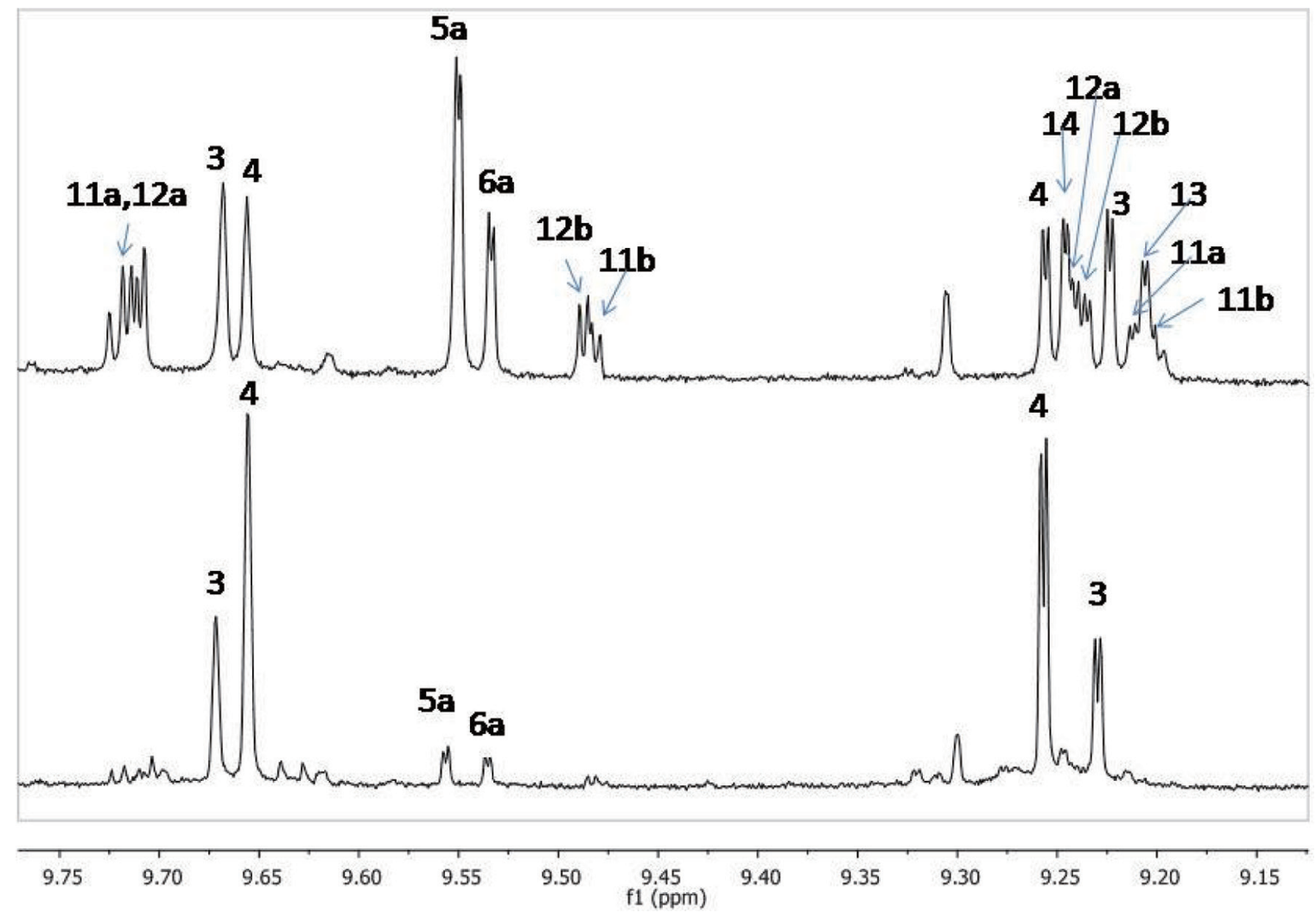

Figure 4. ${ }^{1} \mathrm{H}$ NMR spectrum of the aldehydic region of Mission olive oil extract sample (a: up) and typical Koroneiki (b: down) showing the peaks corresponding to compounds 3-10 (Melliou et al., 2015, 2017).

hydroxytyrosol derivatives and consequently their colorimetric quantification can provide a fast and cheap way to predict whether an oil qualifies for the EU health claim criteria.

\section{Stability of polyphenols: cooking effects}

VOO is considered the best oil for cooking and frying, due to its lipid profile and its phenolic antioxidants. Moreover, the antioxidant activity of vegetables has been shown to increase when they are cooked with VOO (Tresserra-Rimbau and Lamuela-Raventós, 2017). Using VOO for cooking, compared to other oils, is a better choice because of its thermal stability, which is elated to its low level of polyunsaturated fatty acids, and its antioxidants, like carotenes, polyphenols, and tocopherols (Olivero-David et al., 2014). Frying oil temperature usually varies from 160 to $240{ }^{\circ} \mathrm{C}$, with 180 ${ }^{\circ} \mathrm{C}$ being the best choice. Temperature and oxygen are factors that can initiate oxidation, which produces a chemical degeneration of the oil, and an accumulation of free radicals with a potential negative effect on health. The oil resistance to oxidation depends on its fatty acid and antioxidants profile (Franco et al., 2014). VOO obtained from green olives contains more antioxidants. Polyphenols, although sensitive to heat and temperature, are the main compounds responsible for oil stability during frying and VOO rich in phenolic content has been shown to be quite stable during cooking (Olivero-David et al., 2014).

Traditionally, Mediterranean countries are using VOO when cooking vegetables. This provides a better taste to the food, because phenols are transferred to the vegetables, whose antioxidant capacity is enhanced. After the cooking process of sofrito (a special veal dish) polyphenols from tomato, onion, and garlic were detected in the olive oil fraction, especially naringenin, ferulic acid, and quercetin (Rinaldi de Alvarenga et al., 2019). As Figure 5 shows, tomato sauce prepared with the higher proportion of VOO was richer in tyrosol and hydroxytyrosol, indicating that when more olive oil was added, a higher amount of phenolics was transferred to tomato sauce. However, a decrease in the concentration of some tomato flavonoids and cinnamic acids was also observed, while caffeic acid increased.

10. Bioavailability and bioaccessibility of olives and olive oil (OO) polyphenols

The term "bioavailability" was defined as the fraction of an ingested nutrient or compound that reaches the systemic circulation and the specific sites where it can exert its biological action (Yao et al., 2015). The majority of studies regarding the bioavailability of olives and $\mathrm{OO}$ phenolics have focused on hydroxytyrosol, tyrosol, and oleuropein. In this section, in vivo and in vitro studies regarding the bioavailability of olives and olive oil phenolics are discussed.

Although the biological activities of the phenolic compounds present in VOO have been clearly demonstrated, it is difficult to find evidence for the specific role of each component in the beneficial effects of the oil, or for the synergistic activity of a combination of compounds (Lozano-Castellón et al., 2019). The degree to which phenolic compounds are bioavailable (absorbed, metabolized, 


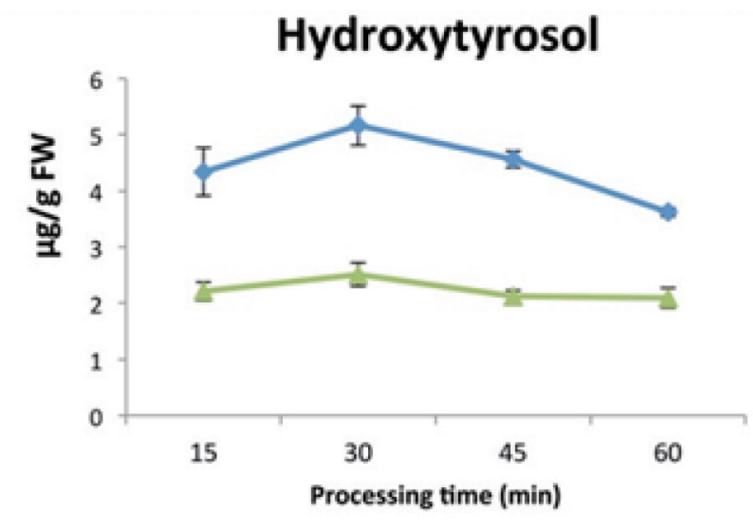

\section{$-5 \%$ Oil content $\rightarrow-10 \%$ oil content}

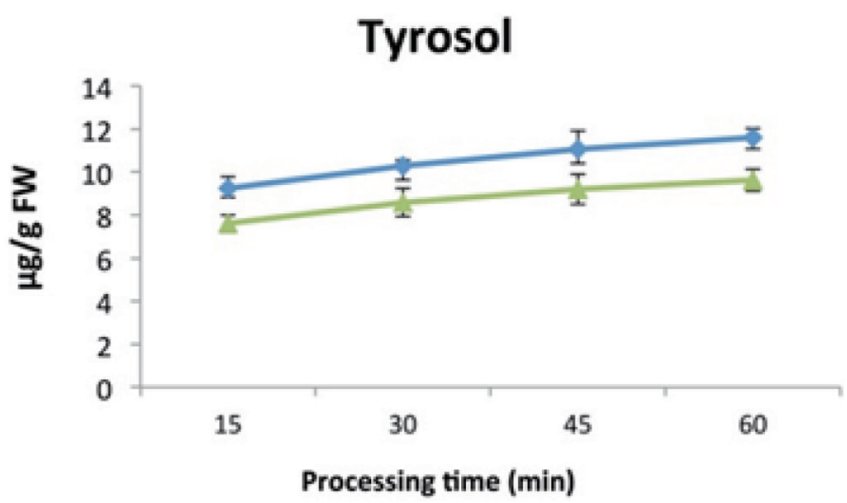

Figure 5. Changes in tomato sauce phenolics from VOO addition over time, using two different concentrations of VOO (5 and $10 \%)$ (Tresserra-Rimbau and Lamuela-Raventós, 2017).

distributed and eliminated) is fundamental in understanding and evaluating the health benefits associated with EVOO consumption in order to achieve an effect in specific tissues or organs (Serreli and Deiana, 2018). Visioli et al. (2000) found that approximately $98 \%$ of hydroxytyrosol and tyrosol from olive oil was absorbed in a dose-response manner. EVOO aglycone secoiridoids can be partially hydrolyzed by the stomach acidic environment releasing free phenolic alcohols; nevertheless a significant amount remains intact and, therefore, gets into the small intestine unmodified.

Although the majority of polyphenols exist in plants as glycosides, in general, only their aglycones can be absorbed in the small intestine, and thus their deglycosylation by $\beta$-glycosidase in epithelial cells is crucial to their absorption (Francisco et al., 2019). The absorption and metabolism of phenolic compounds are determined primarily by their physicochemical characteristics, including molecular size, basic structural properties, polarity, degree of polymerization or glycosylation, solubility, lipophilicity and conjugation with other phenols. The chemical structure of polyphenols, more than the concentration, determines the rate and extent of absorption and the nature of the metabolites circulating in the plasma (Lozano-Castellón et al., 2019). Absorption of polar tyrosol and hydroxytyrosol is thought to occur via passive diffusion, while oleuropein-glycoside, also polar but larger, may be absorbed via a glycose transporter after diffusion through the lipid bilayer of the epithelial cell membrane (Manna et al., 2009). Pinoresinol and 1 -acetoxypinoresinol are quite stable under gastric and duodenal digestion conditions, as proven in vitro with models simulating both conditions (Soler et al., 2010).

According to García-Villalba et al. (2010) once the flavonoid aglycones are absorbed by the intestinal cells, they will undergo an important metabolism by the action of diverse enzymes, mainly transferases. The main conjugated metabolites of flavonoids are glucuronidated forms followed by sulphated and methylated forms. The flavonoids methyl-monogluguronides of apigenin and luteolin have been identified in human urine. The reactions of methylation, glycuronidation and sulfation take place through the respective action of catechol-O-methyl transferases (COMT), uridine-5'-diphosphate glucuronosyl transferases (UDPGT) and sulfotransferases (SULT) (Manach et al., 2004). Glucuronoid conjugates of the polyphenol classes in olive oil have been detected in urine and plasma. A small amount (about 5\%) of ingested olive oil polyphenols are recovered in the urine as glucuronide conjugates of hydroxytyrosol and tyrosol. The rest of them are metabolized into other compounds like 0 -methylated hydroxytyrosol or monosulfate conjugates. Sinse some of these results were obtained only from animal studies, some further research is needed to elucidate the mechanisms of absorption, metabolism and excretion of other key phenolic compounds from olive oil in humans (Gómez-Romero et al., 2012).

The microbial metabolism of phenolic compounds (oleuropein, oleacein and hydroxytyrosol) in the intestinal tract started in small intestine and was intensified in the lower part of the gut (caecum). It detected metabolites derived from oxidation, dehydroxylation, decarboxylation ( $\alpha$-oxidation) and carboxylation reactions along the gastrointestinal tract (López de las Hazas et al., 2016). In past years, EVOO phenols and their metabolites have been evidenced as modulators of gut microbiota composition and function, by suppressing the growth of harmful bacteria and favoring beneficial bacteria (prebiotic-like effects) (Francisco et al., 2019). Phenolics are broken down in colon by macrobiotics and converted to other 
phenolics, which have a notable effect on their bioavailability. Non-absorbed phenolic compounds may also exert antioxidant activity in the gastrointestinal tract, since isolated polyphenols can scavenge free radicals formed by the fecal matrix and the epithelial cells of the intestine (Owen et al., 2004).

\subsection{In vitro digestion and absorption (human and animal stud- ies)}

Experimental, clinical and epidemiological studies have supported the beneficial health effects of olives and olive oil polyphenols which act as antioxidants and radical scavengers with anti-tumor and anti-inflammatory properties (Beauchamp et al., 2005). In particular, their beneficial effects on plasma lipid levels and on the oxidation of LDL cholesterol oxidative damage, as well as on the prevention of oxidative stress resulted in a positive health claim being accepted by the European Food Safety Authority (EFSA, 2011). Tsimidou et al. (2018), in particular, suggested that the health claim EFSA adopted for "olive oil polyphenols" should refer to both tyrosol and hydroxytyosol, free or in bound forms. The pigments chlorophyll and pheophytin and the flavor minor components of olive oil have been shown predominant bioactivity which is related to the facilitation of the absorption of antioxidants present in the oil and in the food to be generally absorbed by our body.

\subsubsection{Human studies}

Direct evidence on bioavailability of olive and olive oil phenolics have been achieved by measuring the concentration of the polyphenols and their metabolites in biological fluids, mainly plasma and urine, after ingestion of pure compounds or olive oil, either pure or enriched with phenolics. Research conducted on humans has shown that hydroxytyrosol and tyrosol are dose-dependently absorbed after ingestion (Covas et al., 2006).

A study conducted by Vissers et al. (2002) demonstrated that absorption of hydroxytyrosol, ligstroside, oleuropein, and tyrosol, was as high as $55-66 \%$. Another study (Miro-Casas et al., 2003) found that even from moderate doses $(25 \mathrm{~mL} / \mathrm{d})$, which is close to that used as daily intake in Mediterranean countries, approximately $98 \%$ of hydroxytyrosol was present in plasma and urine, mainly as glucuronide conjugates, also confirmed by Garcia-Villalba et al. (2010) and Khymenets et al. (2016). Later, hydroxytyrosol sulfate (López de las Hazas et al., 2016), vanillin sulfate (Suarez et al., 2011), and hydroxytyrosol acetate sulfate (Rubio et al., 2012a) were found to be the main biological metabolites of hydroxytyrosol in plasma after olive oil ingestion. In addition, hydroxytyrosol also undergoes the action of catechol-O-methyl transferase, an enzyme involved in the catecholamine catabolism, resulting in the excretion of homovanillic alcohol and homovanillic acid in plasma and urine (Oliveras-Lopez et al., 2014).

It is known that polyphenols are subjected to phase I and phase II metabolism, in which they are hydrolyzed (phase I) and conjugated (phase II) into their glucuronidated, methylated, and sulfated forms in order to be absorbed (Manach et al., 2004). This is a major drawback in understanding the biological activity of hydroxytyrosol in vivo. The picture gets even more complicated by the fact that hydroxytyrosol is also a dopamine metabolite, and body fluid concentrations combine exogenous and endogenous sources (De la Torre, 2008). Overall, the exact absorption mechanism of olive oil phenolics in humans still remains unclear; however, the polarity of the phenolic compounds has been suggested to play an important role. Table 4 shows animal studies regarding the bioavailability of olive and olive oil phenolics in humans.

\subsubsection{Animal studies}

Animal studies showed that phenolic compounds were present in plasma and urine as hydroxytyrosol and tyrosol, mainly as glucuronide and sulfate conjugates, and in methylated form as homovanillic alcohol and homovanillic acid (Kano et al., 2016).

On the other hand, free forms of some phenolic compounds were also reported, such as oleuropein derivative in plasma and brain; luteolin in the kidney, testicle, brain, and heart; or hydroxytyrosol in the plasma, kidney, and testicle (Serra et al., 2012). Furthermore, a recent study demonstrated that the changes in the metabolic disposition of hydroxytyrosol in rats were dose-dependent. In particular, following the treatment of $1 \mathrm{mg} / \mathrm{kg}$ of hydroxytyrosol, approximately $25-30 \%$ of the compounds present in urine were glucuronides, whereas lower recoveries were observed for sulfate conjugates (14\%). However, at higher doses (10-100 mg hydroxytyrosol $/ \mathrm{kg}$ ), sulfates were predominant (57-75\%) (Kotronoulas et al., 2013). In addition, the bioavailability of phenolics of olive oil is shown to depend on the carrier by which they are administered (oil or water), as well as on the administration route (intravenous or oral) (Tuck et al., 2001).

The exact absorption mechanism of olive oil phenolics remains uncertain. However it is proposed that oleuropein-glycoside may diffuse through lipid bilayers of the epithelial cell membrane and be absorbed via a glycose transporter. Additional absorption mechanisms for oleuropein-glycoside are the paracellular route or transcellular passive diffusion (Edgecombe et al., 2000). Recent studies also reported gender differences in plasma and urine metabolites from rats after oral administration of normal and high doses of hydroxytyrosol, hydroxytyrosol acetate, and DOPAC (2,3-DihydrOxyPhenylAceticAcid) (Domínguez-Perles et al., 2017).

\subsubsection{In vitro studies}

Methods for simulating the human digestive tract are being extensively used in vitro, at present, since they are fast, safe, and do not have the same ethical restrictions as in vivo methods (Kamiloglu et al., 2014). The effect of gastric juice on olive oil phenolics has been examined in vitro by incubating the samples at $37^{\circ} \mathrm{C}$ in simulated gastric conditions and during normal physiological time frames (Corona et al., 2006; Soler et al., 2010; Pinto et al., 2011). Although hydrolysis takes place, releasing free phenolics, some fractions of the conjugated forms were still present unhydrolyzed and, thus, entered the small intestine unmodified (Corona et al. 2006). Intestinal digestion of the phenolic compounds from olives and olive oil was simulated by using either enzymatic (Fernández-Poyatos et al., 2019; Quintero-Flórez et al., 2018; Soler et al., 2010) or isolated perfused rat intestines models (Corona et al., 2006; Edgecombe et al., 2000; Pinto et al., 2011). Research showed that $8-90 \%$ of the olive oil phenolics were bioaccessible (Fernández-Poyatos et al., 2019; Quintero-Flórez et al., 2018). On the other hand, oleuropein as well as its aglycone and dialdehydic form are not well absorbed as such in the small intestine (Corona et al., 2006; Edgecombe et al., 2000; Pinto et al., 2011; Soler et al., 2010) the major metabolites detected using the perfused rat intestine model were the glucuronide conjugates of the reduced form of these compounds (Pinto et al., 2011). It is also quite likely that oleuropein reaches the large intestine, where it is subjected to degradation by the colonic microflora (Corona et al., 2006).

Also, in vitro colon fermentation of the olive oil phenolic com- 


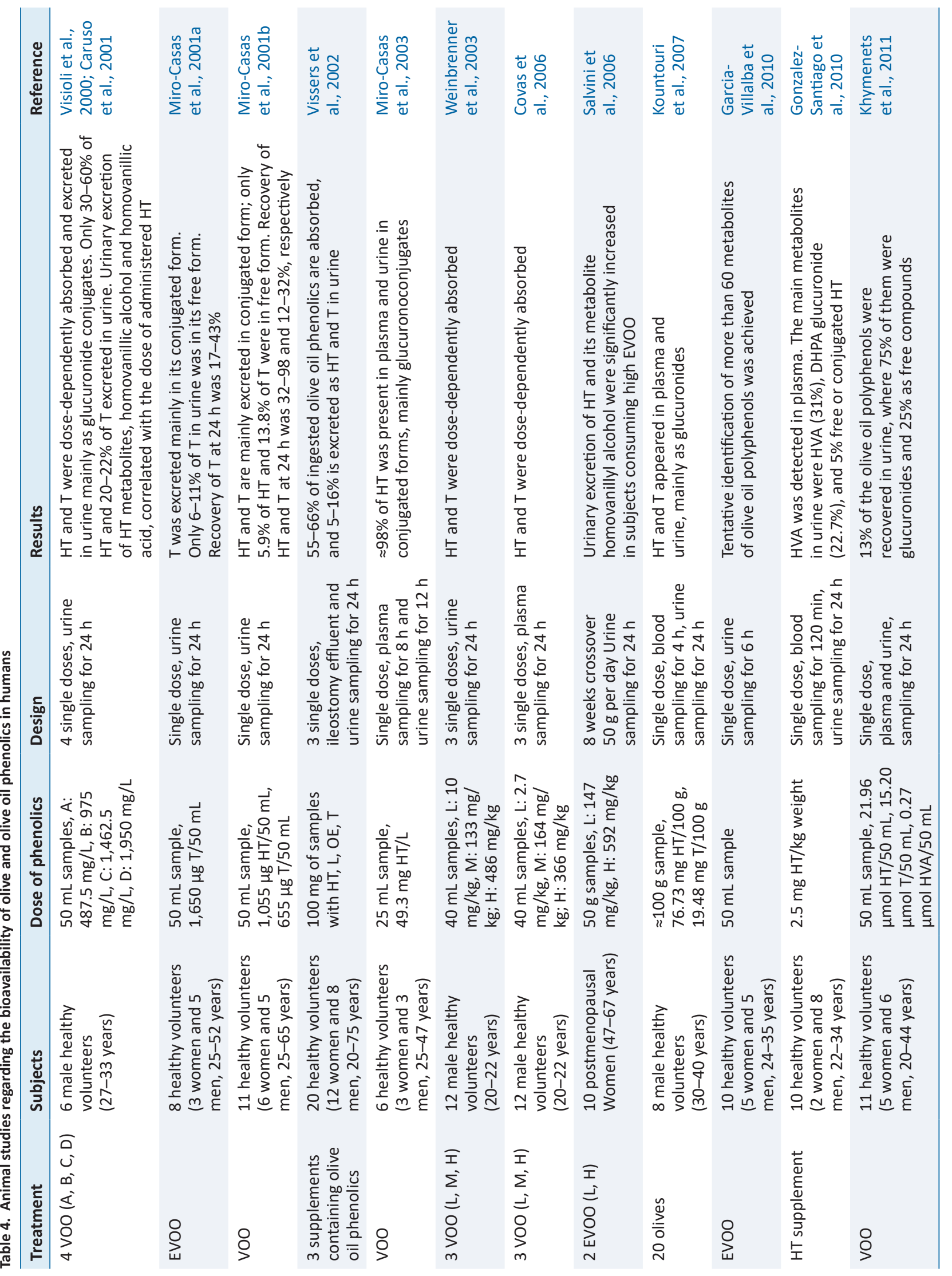




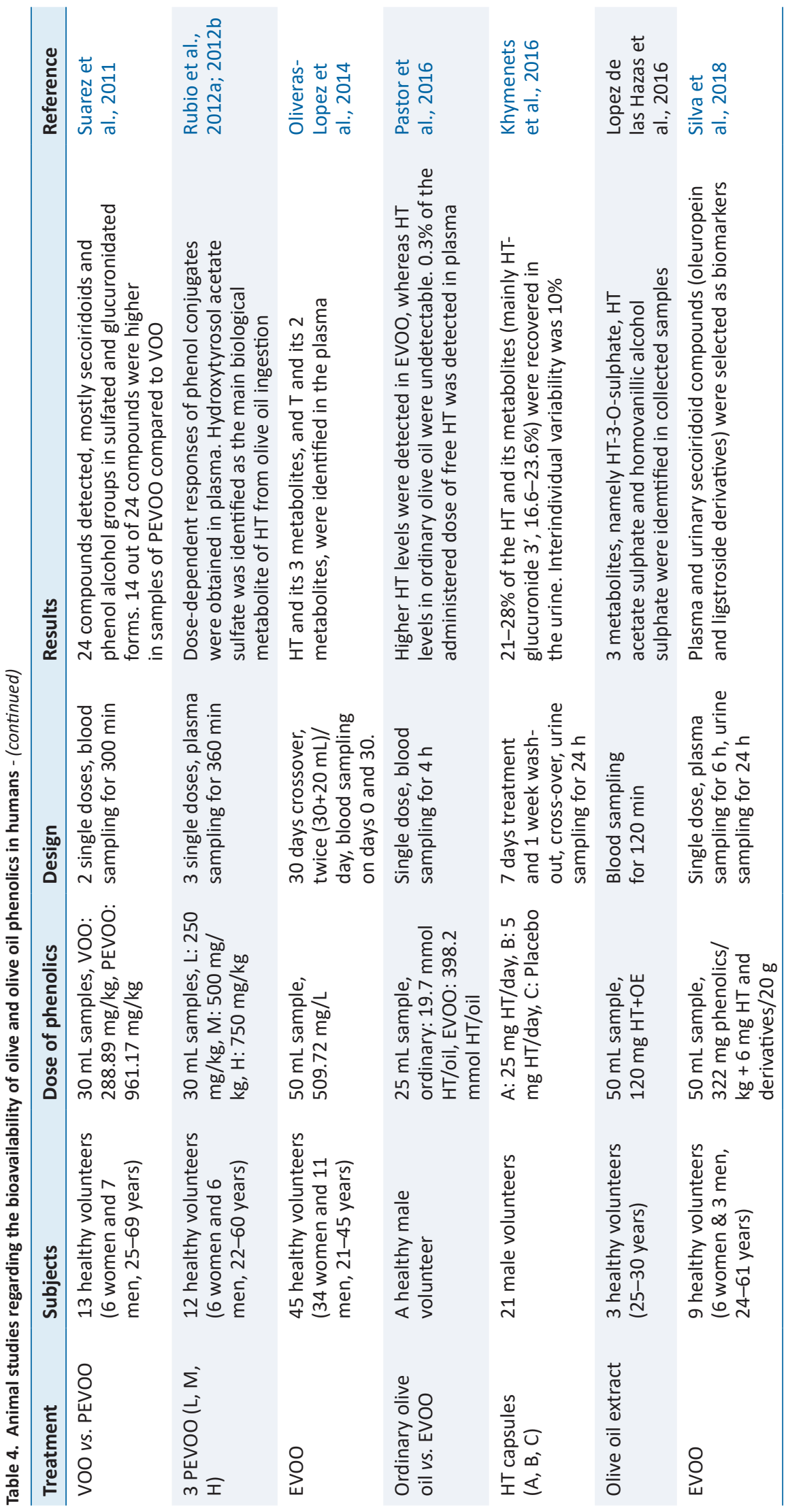




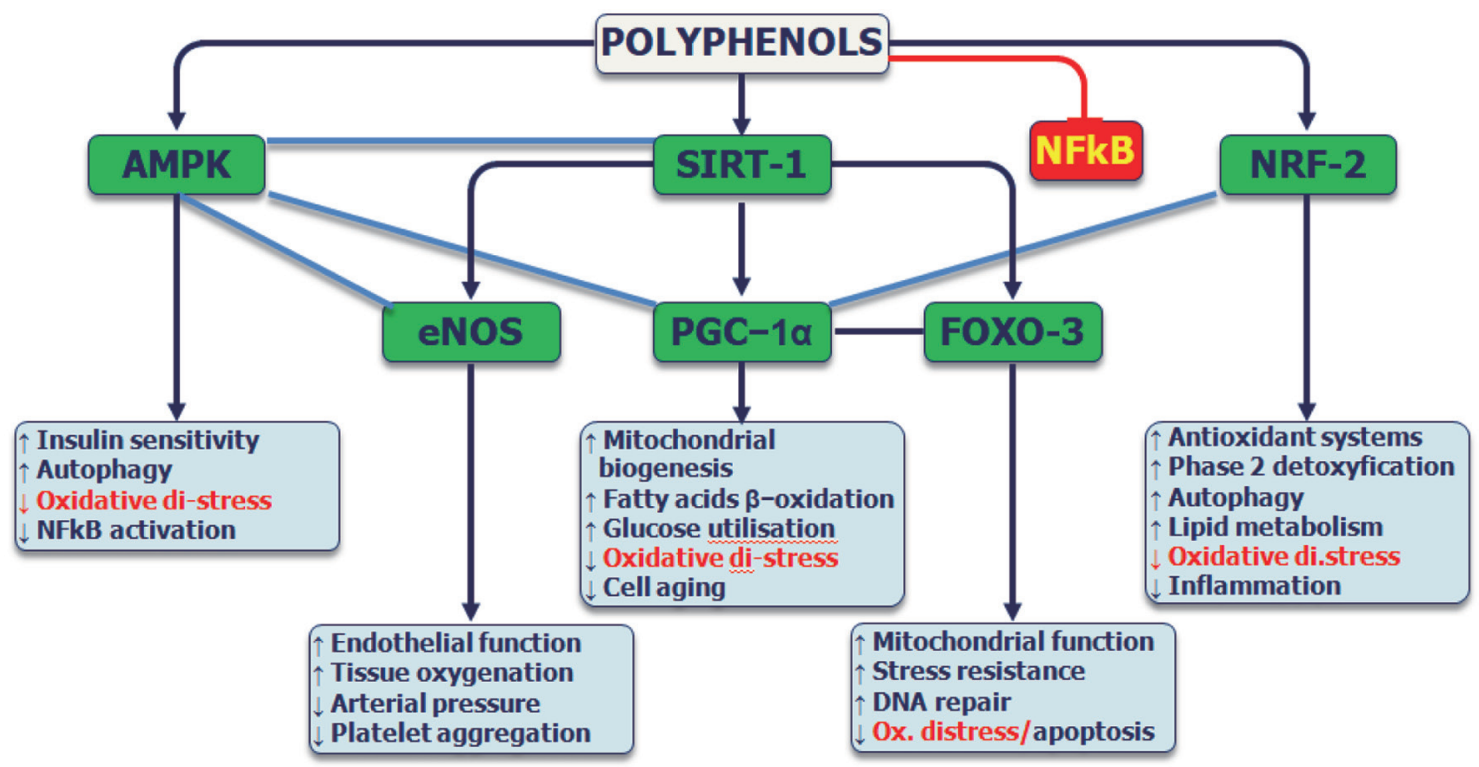

Figure 6. Polyphenols activities in human bodies. Source: Kindly granted by Luigi lorio, E. (2020)

pounds showed an increase in phenolic acids, stability of hydroxytyrosol and tyrosol, as well as high degradation of hydroxytyrosol acetate and oleuropein (Mosele et al., 2014).

\section{Health effects of olives and olive oil polyphenols}

There is an extensive interest for in depth studies in bioactive food substances, in order to evaluate their effects on health, including antioxidant, antiallergic, antimicrobial, antithrombotic, antiatherogenic, hypoglycemic, anti-inflammatory, antitumor, cytostatic, and immunosuppressive properties, and protective activities. Health effects of olive oil because of its high content in oleic acid were always appreciated, but nowadays olive is considered more than just a healthy oil. Consumption of olive oil polyphenols contributes to the protection of blood lipids from oxidative damage. This health claim was approved by the European Food and Safety Administration (EFSA) in 2011. Since the use of health claims in the European Union was harmonized in 2006, only a few foods and ingredients have met the stringent requirements accorded by EFSA. Double-blind, randomized, placebo-controlled trials in humans that demonstrate cause-effect relationships are crucial for approval. Moreover, the effective amount of food (or food constituent) has to be easily consumed in the context of a balanced diet. That is the case of polyphenols in EVOO, since $5 \mathrm{mg}$ of hydroxytyrosol and its derivatives (e.g. oleuropein complex and tyrosol) can be consumed daily (approximately two tablespoons of oil) in order to obtain the desired benefits (EFSA Panel on Dietetic Products, 2011). Nevertheless, the health benefits of olive oil polyphenols go far beyond those related to the health claim.

Generally, phenolic compounds have been described to inhibit the induction of oxidation by metals, scavenge free radicals, act as reductants and even preserving the flavor of foods, e.g., virgin olive oil. In addition, olive oil biophenols have been shown to act as antimicrobial agents, which suggests their use as a natural mean to increase the shelf life of foods (Caporaso et al., 2018). Phenolic compounds in olive fruit significantly influence the health benefits and sensory properties of processed olive oil and table ol- ives (Crawford et al., 2018). Oleocanthal is described as the major EVOO phenolic compound with functional and health benefits, including anti-inflammatory, chemotherapeutic, neuroprotective, and antirheumatic (Francisco et al., 2019). In addition, every year new data point to oleacein as the main phenol responsible for the positive effect of olive oil on cardiovascular disease. This compound also displays a protective effect against atherosclerosis and oxidation, and anti-inflammatory activity (Lozano-Castellón et al., 2019). Figure 6 shows the activity of polyphenols in human bodies.

\subsection{Protection against oxidative damage and inflammation}

Reactive oxygen species (ROS) are chemically reactive molecules produced by the normal metabolism of organisms. Under environmental stress, the level of ROS increases and can damage lipids, DNA and proteins. Besides oxidative damage, ROS activate proand anti-inflammatory cytokines (Valko et al., 2007). The beneficial properties of hydroxytyrosol are strongly related to its ability to scavenge free radicals and reactive oxygen/nitrogen species as well as to activate endogenous antioxidant systems in the body (Gorzynik-Debicka et al., 2018). The anti-inflammatory activity of oleocanthal has been correlated with its inhibitory effect on COX1 and COX-2, enzymes responsible for producing inflammatory mediators such as prostaglandins and thromboxane. The healthpromoting activities of oleacein include metal ion chelation and anti-inflammatory activity through COX-2 inhibition (LozanoCastellón et al., 2019).

EVOO polyphenols are well-known for their anti-inflammatory effects, exerted by down-regulating inflammatory mediators by transcriptional or post-transcriptional mechanisms and by modulating the activation of kinases involved in the onset of inflammatory process at different levels (Serreli and Deiana, 2018). Higher concentrations of pro-inflammatory agents in serum, like thromboxane B2 (TXB2) and leukotriene B4 (LTB4), are known to be risk factors for cardiovascular diseases. Some investigators noticed that TXB2 and LTB4 concentrations decrease in a dose-response 
manner,with the phenolic concentration of olive oil (Covas et al., 2006). Other pro-inflammatory agents, such as interleukin-6 (IL-6) and C-reactive protein (CRP), also decreased after consumption of olive oil in a placebo crossover randomized trial with coronary heart patients (Fitó et al., 2008).

\subsection{Cardiovascular diseases, $L D L, H D L$ and endothelial func- tion}

Olive oil polyphenols decrease the cases of cardiovascular diseases by acting on their main risk factors. Apart from decreasing oxidized LDL cholesterol (Castañer et al., 2012), they increase HDL particles (Hernáez et al., 2014) and HDL anti-inflammatory activity (Loued et al., 2013). It is possible that metabolic activity of olive oil polyphenols is related to their capacity to bind LDL and decrease oxidation in these particles. This is important since oxidized LDL (oxLDL) plays a basic role in the development of atherosclerosis.

According to Castañer et al. (2012), polyphenols from olive oil reduced LDL oxidation by scavenging free radicals and by modulating gene expression. More specifically polyphenol-rich olive oil reduced CD40L gene expression, down-stream products, and related genes involved in atherogenic and inflammatory processes in humans. Besides, the effects on plasma lipids and endothelial function, some authors reported a beneficial effect of polyphenols on blood pressure (Castañer et al., 2012).

The final results of the PREDIMED study concluded that Mediterranean diets reduced the incidence of major cardiovascular events (stroke, myocardial infarction and cardiovascular death) by approximately $30 \%$. The multivariable-adjusted hazard ratios (HRs) were $0.70,0.54$ to 0.92 and $0.72,0.54$ to 0.96 for the group assigned to a Mediterranean diet with extra-virgin olive oil and the group assigned to a Mediterranean diet with nuts, respectively, versus the control group (Estruch et al., 2013). Within the PREDIMED trial, an observational prospective cohort study found that EVOO consumption was associated with reduced cardiovascular risk. Total olive oil consumption was also significantly associated with $48 \%$ reduced risk of cardiovascular mortality. For each 10 $\mathrm{g} / \mathrm{d}$ increase in EVOO consumption, cardiovascular disease and mortality risk decreased by 10 and 7\%, respectively (Guasch-Ferré et al., 2014).

In a post-hoc analysis of the same trial, EVOO in the context of a Mediterranean dietary pattern significantly reduced the risk of a trial fibrillation (HR $0.62 ; 0.45-0.85$ ) compared with the low-fat diet group, whereas no effect was found for the Mediterranean diet with nuts, suggesting the important role of olive oil in the prevention of arrhythmias (Martínez-González et al., 2014). These findings are supported by other prospective cohort studies. In the Three-City Study in France, high OO consumption and high plasma oleic acid as an indirect biological marker of OO intake were associated with lower incidence of stroke in older subjects (Samieri et al., 2011). Within the EPICOR (long-term follow-up of antithrombotic management patterns in acute coronary syndrome patients) study, a strong inverse association was found between increasing consumption of olive oil and coronary heart disease risk (Bendinelli et al., 2011). Similarly, it was found that OO was associated with a reduced risk of overall mortality, CVD mortality and incident coronary heart disease events in a large Mediterranean cohort within the EPIC-Spain (The European Prospective Investigation into Cancer and Nutrition) study (Buckland et al., 2012).

As mentioned by Hernáez et al. (2014), olive oil polyphenols promote the main HDL antiatherogenic function, its cholesterol efflux capacity. These polyphenols increased HDL size, promoted a greater HDL stability reflected as a triglyceride-poor core, and enhanced the HDL oxidative status, through an increase in the olive oil polyphenol metabolites content in the lipoprotein. They suggested that the main biological function of the HDL is extracting the excess of cholesterol from the peripheral cells and taking it to the liver to be metabolized and excreted. This cholesterol capture is broadly known as cholesterol efflux, and the main cell types involved in it are the macrophages under the endothelium in blood vessels (Yancey et al., 2003). This HDL functional property has been broadly tested in several macrophage cell lines and has been inversely related to early atherosclerosis development and to high risk of experiencing a coronary event (Khera et al., 2011). The consumption of olive oil polyphenols enhanced the cholesterol efflux capacity of the volunteers' HDL in a physiological model of THP1 monocyte-derived macrophages. A similar effect has been described after EVOO consumption in a noncontrolled, linear study, after walnuts consumption (Berryman et al., 2013) or a pioglitazone treatment (Khera et al., 2011).

The HDL cholesterol efflux enhancement after the HPCOO intervention may be promoted by the observed increase in the olive oil polyphenol metabolites bound to the lipoprotein. It has been described by Bonnefont-Rousselot et al., (1995) that an oxidized HDL is more rigid and presents a lower cholesterol efflux capacity. Thus, a better antioxidative protection, conferred by a higher content of olive oil polyphenols in the HDL, may contribute in understanding its functional enhancement. As observed by Tuck et al. (2002), the olive oil polyphenol metabolites bound to the HDL after the HPCOO intervention have been shown to have antioxidant properties. Thus, a local antioxidant effect on the HDL is expected. In parallel, in the HPCOO intervention, a less rigid HDL was observed, and an increased HDL fluidity is considered an intermediate marker of enhanced HDL functionality. Taking evidence into consideration, it can be hypothesized that the binding of olive oil polyphenols to HDL would increase the fluidity of the particle, thus enhancing the HDL capacity for promoting the cholesterol efflux from cells (Hernáez et al., 2014).

According to Otvos et al., (2006), the only HDL functional parameter that has been correlated to incident coronary events is the HDL particle number. So it was assessed by Hernáez et al. (2014) whether positive changes in this direction happened after the consumption of olive oil polyphenols. Although nonsignificant, a trend toward higher HDL particle count values after the HPCOO intervention was observed. Moreover, a direct relation between baseline values of this parameter and cholesterol efflux capacity was found. However, as these results are not conclusive, and because few treatments have been able to induce an enhancement in HDL particle count values, further studies in this line are priority to check this hypothesis.

There are findings, nowadays, that triglyceride levels in the HDL core are present in several cardiovascular pathologies, such as coronary heart diseases (Syvänne et al., 1995) and they are related to a less stable conformation of apolipoprotein-A1 in HDL surface (ie, a less stable lipoprotein structure). It was found that significantly lower triglyceride levels in the HDL core were observed. This decrease, according to Kontush et al., (2006), could be explained by a lower activity of the cholesterol ester transfer protein (CETP). CETP extracts esterified cholesterol from the HDL core to triglyceride-rich lipoproteins, returning triglycerides from triglyceride-rich lipoproteins to HDL. Lam et al., (2008) suggest that this could have happened through a direct blockade of CETP enzyme, as it has been described for apple polyphenols, or through a nutrigenomic inhibition of CETP gene expression, similar to the one reported after a Mediterranean diet consumption (Ros et al., 2014) 
Covas and Gaddi, (2011), in their letter to "The Journal of Nutrition" editor mentioned that most bioavailability studies conducted so far agree that plasma and tissues concentration of EVOO polyphenols metabolites is often higher than the concentration reached by the ingested parent compounds. Thus, these metabolites are likely to significantly contribute to the beneficial health effect correlated to the regular consumption of olive products.

\subsection{Diabetes and obesity}

Basterra-Gortari et al. (2019) stated that a healthful eating pattern, such as the Mediterranean eating plan (Med-EatPlan), is a key component of type 2 diabetes management. The traditional Mediterranean pattern is characterized by a high intake of olive oil, fruits, vegetables, nuts, and cereals; a moderate intake of fish and poultry; a low intake of red meat, whole-fat diary, and sweet desserts; and wine consumption with meals is allowed in moderation. In summary, our study results show that PREDIMED participants with type 2 diabetes who underwent an intervention with an energy-unrestricted Med-EatPlan+EVOO had significantly lower rates of initiation of glycose-lowering medications.

EVOO consumption in a regular basis, especially as an animal fat replacer, is a safe way to manipulate postprandial blood glycose response after high-glycemic index meal in type 1 diabetes patients, through complex interactions between the macronutrient composition of the meal and gastro-intestinal sensing that involve gastric emptying, incretins secretion, and lipid metabolism (Bozzetto et al., 2019).

According to Wijayanthie et al., (2019), MUFA cause changes in the composition of cell membranes so that they are richer in cistype fatty acids. This alteration of composition results in the formation of more spaces between membrane head groups so that they are more broadly hydrophilic. This change increases the fluidity of cell membranes and activates key receptor proteins (G proteins, protein kinase $\mathrm{C} \alpha$ subunits), which can reach the membrane surface readily and increase signal sensitivity. MUFAs also improve the entero-insular axis by increasing the secretion and activity of glucagon-like peptide (GLP)-1 and gastric inhibitory polypeptide. This increase can increase the secretion and biosynthesis of insulin. In addition, GLP-1 can reduce glucagon levels, which makes GLP-1 effective as nutritional therapy in DM. MUFAs also reduce damage and trigger neogenesis of pancreatic beta cells.

Santangelo et al. (2016) suggested that both fasting plasma glycose and $\mathrm{HbAlc}$ significantly decreased after HPCOO intake. In addition, ingestion of HPCOO resulted in the reduction in body weight and BMI. In addition, HPCOO consumption determined a reduction in serum levels of AST, ALT. As for lipid profile, decreased HDL level was observed after HPCOO consumption, without modifying the total cholesterol/HDL (TC/HDL), LDL/ HDL and triglycerides/HDL ratios. No changes were observed in the other assessed variables after EVOO ingestion. The main finding of the present study is that the consumption of HPCOO improves anthropometric (body weight and BMI) and biochemical parameters (fasting glycose, HbAlc, liver enzymes and visfatin) in patients with T2D.

This research also provided the first evidence about the effect of HPCOO consumption on visfatin in humans, in agreement with those of other studies, which have shown an association between $\mathrm{MD}$ and a significant improvement in metabolic control in patients with T2D diabetes (Violi et al., 2015; Visioli et al., 2001). In particular, MD supplemented with extra-virgin olive oil strongly reduces the risk of T2D in Mediterranean population at high risk of CVD and downregulates the expression of atherosclerosis-related genes in peripheral blood mononuclear cells, in healthy subjects. Moreover, higher olive oil intake has been associated with a reduced T2D risk in a large US women population as well.

In the light of the above findings, they stated that the changes found in metabolic control parameters drove them into the conclusion that EVOO polyphenols can have a crucial role in these effects, since they represent the only difference in composition of EVOO compared to ROO. Weight loss and inflammatory status improvement have been observed in overweight subjects following diet and physical activity modifications, and adherence to MD has been linked to a lower rate of obesity. After HPCOO ingestion, a reduced BMI in the subjects was observed even though the diet was the same throughout the intervention period.

Regular EVOO consumption and its effect on obesity was also researched by Cândido et al. (2018). Their study was designed to assess the effects of EVOO incorporated into an energy-restricted non-Mediterranean diet program on body weight, body composition and metabolic biomarkers in women with excess body fat. The main finding of the study was that the consumption of EVOO increased total fat loss and reduced diastolic blood pressure compared to the control soybean oil group.

A clinical trial conducted in 17 type 1 obesity participants (BMI between 30.1 and $33.3 \mathrm{~kg} / \mathrm{m}^{2}$ ) with a polyphenol supplement of $370 \mathrm{mg}$ of total polyphenols by Guo et al. (2017), showed a significant reduction in body weight, BMI, and waist and hip circumference compared with a placebo group after 12 weeks of intervention. A study conducted by Marranzano et al. (2018) demonstrated that higher dietary intake of flavonoids is inversely associated with an excess of weight and obesity. A longitudinal study that included 4,280 participants aged 55-69 years showed an association between a higher flavonoids intake and a lower increase in BMI in women (Wang et al., 2014).

Regarding EVOO polyphenols, Vogel et al. (2015) suggested that tyrosol derivates, such as oleuropein, are involved in energy metabolism and adiposity, improving insulin sensitivity. Another interesting mechanism studied by Oi-Kano et al. (2017) in experimental models showed an increase in uncoupling protein 1 (UCP1) expression, which translates to the formation of "beige" adipose tissue, leading to a decrease of visceral fat mass.

\subsection{Protection against cancer}

Many epidemiological studies highlight that a Mediterranean diet decreases the risk of some types of cancer, especially those of the digestive tract (Gotsis et al., 2015; Grosso et al., 2013). This probably derives from high consumption of olive oil in Mediterranean countries. A meta-analysis of 19 observational studies came to the conclusion that a higher intake of olive oil was associated with lower risks of having any type of cancer (Psaltopoulou et al., 2011).

Reduction of environmental and food carcinogen bioavailability, protection against oxidative stress, inhibition of enzymes related to tumor promotion and metastasis, a direct effect on nucleic acids and nucleoproteins and interactions with proteins controlling cell cycle progression and gene expression were some of the mechanisms proposed to explain the anti-cancer properties of olive oil (Barbaro et al., 2014; Cárdeno et al., 2013; Martín-Peláez et al., 2013; Serreli and Deiana, 2018). The chemopreventive activity of olive oil has been attributed to hydroxytyrosol, tyrosol oleuropein aglycon, ligstroside aglycon, oleocanthal and oleuropein. The animal studies have confirmed the ability of these compounds to inhibit the carcinogenesis process at both initiation and promotion/progression stages. The human intervention trials, on DNA damage, have shown a significant preventive effect on oxidative 
DNA damage in terms of reduction of 8-oxo-7,8-dihydro-2'deoxyguanosine in urine, in mitochondria DNA of mononuclear cells and in lymphocyte DNA (Fabiani, 2016).

\subsection{Neuroprotective effect}

There is evidence about the relation between olive oil and neurodegenerative diseases. In vitro, neuroprotective activities attributed to phenolic compounds from VOO include interference with amyloid and tau protein aggregation, and reduction of $\mathrm{A} \beta$ deposition, production, and induced inflammation, as well as enhanced $A \beta$ clearance, decreased inflammatory biomarkers, oxidative stress, and apoptosis, lowering the cerebral infarct volume and damage after induced injury, and attenuation of insulin resistance, mitochondrial dysfunction, and ATP depletion. These compounds are able to protect against cerebral ischemia, spinal cord injury, Huntington's disease, and Alzheimer's disease. However, many of the mechanisms behind these protective actions have not been fully explained (Khalatbary, 2013).

Studies in animals revealed that oleuropein and hydroxytyrosol play an important role in preventing or attenuating Parkinson's and Alzheimer's diseases. For instance, oleuropein counteracts amyloid plaque generation and deposition, and inhibits Tau aggregation, which are characteristics of Alzheimer's disease (Carito et al., 2014; Sarbishegi et al., 2014). Other authors also examined the effects of EVOO on learning and memory in SAMP8 mice, an age-related learning/memory impairment model associated with increased amyloid- $\beta$ protein and brain oxidative damage. Their findings suggested that EVOO has beneficial effects on learning and memory deficits related to the overproduction of amyloid- $\beta$ protein by reversing oxidative damage in the brain (Farr et al., 2012). Results from intervention studies about olive oil and neuroprotection are very scarce, since most of the available studies were performed in experimental animals. However, a long-term intervention with a Mediterranean diet supplemented with EVOO resulted in a better cognitive function in comparison with a low-fat diet in a subsample of the PREDIMED trial (Martínez-Lapiscina et al., 2013). Hydroxytyrosol is even capable of crossing the bloodbrain barrier. However, data in this field are still very limited and further investigation is needed. According to an in vitro study with human retinal pigment epithelial cells (ARPE-19), age-related macular degeneration, which in most cases leads to blindness, can also be prevented with hydroxytyrosol (Zhu et al., 2010).

\subsection{Other effects}

Results with olive oil polyphenols such as oleuropein, hydroxytyrosol and tyrosol have demonstrated in vitro potent antimicrobial activity against several strains of bacteria responsible for intestinal and respiratory infections (Barbaro et al., 2014). Olive oil polyphenols can improve the human macrobiotics as well, since most of them reach the lower parts of the gastrointestinal tract, where they can be metabolized.(Martín-Peláez et al., 2013). Oleuropein was found to be effective against various strains of bacteria, viruses, fungi, molds and even parasites (Gorzynik-Debicka et al., 2018). Moreover it possesses prebiotic properties, since Lactobacillus and Bifidobacterium strains may utilize it as a carbon source and exhibits a significant antimicrobial activity against Clostridium and E. Coli (Deiana, 2018). As far as hepatic dysfunction is concerned, olive oil and its phenolic compounds has been associated with the modulation of hepatic lipid metabolism, including protective effects against steatosis, lipid synthesis, and endoplasmic reticulum stress, as well as induction of antioxidant/detoxicant enzymes, mitochondrial biogenesis, and mitochondrial function. In a clinical study done with healthy subjects, in the frame of a Mediterraneanstyle diet, EVOO improved post-prandial glycose and LDL-cholesterol levels. A higher olive oil intake has been associated with a modestly lower risk of type-2 diabetes in women from a US cohort and this agrees with the previous results (Guasch-Ferré et al., $2015)$. Other in vitro and in vivo studies also showed the relation of olive oil and its bioactive compounds with hepatoprotection, a decrease in chronic inflammatory disorders, nutrigenomic effects, and bone health (Barbaro et al., 2014; Martín-Peláez et al., 2013). For all these to be confirmed more studies especially in humans, are required.

\section{Conclusions}

VOO, due to its lipid profile and rich phenolic composition, has unique sensory and health properties. The complexity of the phenolic profile of olives and VOO requires mass spectrometry or nuclear magnetic resonance spectroscopy for its determination. Initially, the properties of olives and VOO were attributed to their high antioxidant levels, but it has become clear that multiple mechanisms are involved, including an impact on gut macrobiotics that may have protective effects against CVD and cancer neurological diseases.

Phenolic compounds in olive fruit are receiving increased attention due to their influence on sensory characteristics (flavor, colour etc.), nutritional properties and scientific evidence of positive health effects. EVOO is one of the most appreciated fat products of the Mediterranean diet, and many positive nutritional properties have been associated with its consumption. The popularity of EVOO is linked both to its health properties, sensory properties, antioxidant activity and its nutritional and functional properties. While its health properties are attributed to phenolic compounds, which are also responsible for its bitterness and pungency, its pleasant aroma is due to the presence of volatile aroma compounds olives, virgin olive oil have attracted a great interest among scientists due to their valuable compounds with health promoting benefits. These benefits mainly arise from the positive bioactive properties of their phenolic compounds (phenolic acids and alcohols, secoiridoid derivatives, lignans and flavones) which have been proven antiviral, antimicrobial, antioxidant, anti-inflammatory and anti-carcinogenic activity.

Several studies have been applied in vivo (to humans and animals) and in vitro (via gastrointestinal digestion studies and cell culture studies) to determine the bioavailability of olives and olive oil phenolics. More studies, however, should be taken into consideration and the methodologies should be adopted accordingly.

\section{References}

Achak, M., Hafidi, A., Ouazzani, N., Sayadi, S., and Mandi, L. (2009). Low cost biosorbent "banana peel" for the removal of phenolic compounds from olive mill wastewater: Kinetic and equilibrium studies. J. Hazard. Mater. 166: 117-125.

Allouche, N., Fki, I., and Sayadi, S. (2004). Toward a high yield recovery of antioxidants and purified hydroxytyrosol from olive mill wastewaters. J. Agric. Food Chem. 52: 267-273.

Al-Malah, K., Azzam, M., and Abu-Lail, N.I. (2000). Olive mills effluent (OME) wastewater post-treatment using activated clay. Sep. Purif Technol. 20: 225-234.

Alowaiesh, B., Singh, Z., Fang, Z., and Kailis, S.G. (2018). Harvest time im- 
pacts the fatty acid compositions, phenolic compounds and sensory attributes of Frantoio and Manzanilla olive oil. Scientia Horticulturae 234: 74-80.

Angerosa, F., Mostallino, R., Basti, C., and Vito, R. (2000). Virgin olive oil odor notes: their relationship with volatile compounds from the lipoxigenase pathway and secoiridoid compounds. Food Chem. 68 283-287.

Bajoub, A., Medina-Rodriguez, S., Gomez-Romero, M., Ajal, E., BagurGonzalez, M.G., Fernandez-Gutierrez, A., and Carrasco-Pancorbo, A (2017). Assessing the varietal origin of extra-virgin olive oil using liquid chromatography fingerprints of phenolic compound, data fusion and chemometrics. Food Chem. 215: 245-255.

Barbaro, B., Toietta, G., Maggio, R., Arciello, M., Tarocchi, M., Galli, A., and Balsano, C. (2014). Effects of the olive-derived polyphenol oleuropein on human health. Int. J. Mol. Sci. 15: 18508-18524.

Basterra-Gortari, F.J., Miguel Ruiz-Canela, M., Martnez-Gonzalez, M.A., Babio, N., Sorlı, J.V., Fito, M., Ros, E., Gomez-Gracia, E., Fiol, M., Lapetra, J., Estruch, R., Serra-Majem, L., Pinto, X., Gonzalez, J.L., Bullo, M., Castaner, O., Alonso-Gomez, A., Forga, L., and Aros, F. (2019). Effects of a Mediterranean Eating Plan on the Need for Glucose-Lowering Medications in Participants With Type 2 Diabetes: A Subgroup Analysis of the PREDIMED Trial. Diabetes Care 42: 1390-1397.

Bazzu, G., Molinu, M.G., Dore, A., and Serra, P.A. (2017). Microdialysis as a New Technique for Extracting Phenolic Compounds From Extra Virgin Olive Oil. J Agric Food Chem 65(8): 1829-1835.

Beauchamp, G.K., Keast, R.S.J., Morel, D., Lin, J., Pika, J., Han, Q., Lee, C.-H., Smith, A.B., and Breslin, P.A.S. (2005). Phytochemistry: ibuprofen-like activity in extra-virgin olive oil. Nature 437: 45-46.

Ben Hasine, K., El Riachy, M., Taamalli, A., and Kiritsakis, A. (2013). Consumer discrimination of Chemlali and Arbequina olive oil cultivars according to their cultivar, geographical origin, and processing system. Eur. J. Lipid Sci. Technol. 116(7): 812-824.

Bendinelli, B., Masala, G., Saieva, C., Salvini, S., Calonico, C., Sacerdote, C., Agnoli, C., Grioni, S., Frasca, G., Mattiello, A., Chiodini, P., Tumino, R., Vineis, P., Palli, D., and Panico, S. (2011). Fruit, vegetables, and olive oil and risk of coronary heart disease in Italian women: the EPICOR Study. Am. J. Clin. Nutr. 93: 275-283.

Bendini, A., Cerretani, L., Carrasco-Pancorbo, A., Gómez-Caravaca, A.M. Segura-Carretero, A., Fernández-Gutiérrez, A., and Lercker, G. (2007). Phenolic Molecules in Virgin Olive Oils: a Survey of Their Sensory Properties, Health Effects, Antioxidant Activity and Analytical Methods. An Overview of the Last Decade. Molecules 12: 1679-1719.

Berryman, C.E., Grieger, J.A., West, S.G., Chen, C.Y., Blumberg, J.B., Rothblat, G.H., Sankaranarayanan, S., and Kris-Etherton, P.M. (2013). Acute consumption of walnuts and walnut components differentially affect postprandial lipemia, endothelial function, oxidative stress, and cholesterol efflux in humans with mild hypercholesterolemia. J. Nutr 143: 788-794.

Bertin, L., Ferri, F., Scoma, A., Marchetti, L., and Fava, F. (2011). Recovery of high added value natural polyphenols from actual olive mill wastewater through solid phase extraction. Chem. Eng. J. 171: 1287-1293.

Bianchi, G., and Pocci, N. (1994). 3,4-Dihydroxyphenylglycol, a major C6C2 phenolic in Olea europaea fruits. Phytochemistry 35: 1335-1337.

Bianco, A., Buiarelli, F., Cartoni, G., Coccioli, F., Muzzalupo, I., Polidori, A., and Uccella, N. (2001). Analysis by HPLCMS/MS of biophenolic components in olives and oils. Anal. Lett. 34: 1033-1051.

Blekas, G., Psomiadou, E., Tsimidou, M., and Boskou, D. (2002). On the importance of total polar phenol measurement for monitoring the stability of Greek virgin olive oils. European Journal of Lipid Science 104: 340-346.

Bonnefont-Rousselot, D., Motta, C., Khalil, A.O., Sola, R., La Ville, A.E., Delattre, J., and Gardès-Albert, M. (1995). Physicochemical changes in human high-density lipoproteins (HDL) oxidized by gamma radiolysis-generated oxyradicals. Effect on their cholesterol effluxing capacity. Biochim Biophys Acta. 1255: 23-30.

Borges, T.H., Lopez, L.C., Pereira, J.A., Cabrera-Vique, C., and Seiquer, I. (2017). Comparative analysis of minor bioactive constituents (CoQ(10), tocopherols and phenolic compounds) in Arbequina extra virgin olive oils from Brazil and Spain. J. Food Compost. Anal. 63 47-54.

Bouaziz, M., Fki, I., Jemai, H., Ayadi, M., and Sayadi, S. (2009). Effect of storage on refined and husk olive oils composition: Stabilization by addition of natural antioxidants from Chemlali olive leaves. Food Chem. 108: 253-262

Bozzetto, L., Alderisio, A., Clemente, G., Giorgini, M., Barone, F., Griffo, E., Costabile, G., Vetrani, C., Cipriano, P., Giacco, A., Riccardi, G., Rivellese, A.A., and Annuzzi, G. (2019). Gastrointestinal effects of extravirgin olive oil associated with lower postprandial glycemia in type 1 diabetes. Clin. Nutr. 38: 2645-2651.

Brenes, M., Garcia, A., Rios, J.J., García, P., and Garrido, A.F. (2002). Use of 1-acetoxypinoresinol to authenticate Picual olive oils. Inter. J. Food Sci. Technol. 37: 615-625.

Britton, J., Davis, R., and O'Connor, K.E. (2019). Chemical, physical and biotechnological approaches to the production of the potent antioxidant hydroxytyrosol. Appl. Microbiol. Biotechnol. 103: 5957-5974.

Buckland, G., Mayén, A.L., Agudo, A., Travier, N., Navarro, C., Huerta, J.M., Chirlaque, M.D., Barricarte, A., Ardanaz, E., Moreno-Iribas, C., Marín, P., Quirós, J.R., Redondo, M.L., Amiano, P., Dorronsoro, M., Arriola, R., Molina-Montes, E., Sánchez, M.J., and Gonzalez, C.A. (2012) Olive oil intake and mortality within the Spanish population (EPICSpain). J. Clin. Nutr. 96: 142-149.

Bulotta, S., Celano, M., Lepore, S.M., Montalcini, T., Pujia, A., and Russo, D. (2014). Beneficial effects of the olive oil phenolic components oleuropein and hydroxytyrosol: focus on protection against cardiovascular and metabolic diseases. Journal of Translational Medicine 12: 219

Cândido, F.G., Valente, F.X., Da Salva, L.E., Coelho, O.G.L., Do Carmo, M.G.P., and De Cassia, R.G.A. (2018). Consumption of extra virgin olive oil improves body composition and blood pressure in women with excess body fat: a randomized, double-blinded, placebo-controlled clinical trial. Eur. J. Nutr. 57: 2445-2455.

Canepa, P., Marignetti, N., Rognoni, U., and Calgari, S. (1988). Olive mills wastewater treatment by combined membrane processes. Water Res. 22: 1491-1494.

Caporaso, N., Formisano, D., and Genovese, A. (2018). Use of phenolic compounds from olive mill wastewater as valuable ingredients for functional foods. Crit. Rev. Food Sci. Nutr. 58: 2829-2841.

Cárdeno, A., Sánchez-Hidalgo, M., and Alarcón-de-la-Lastra, C. (2013). An up-date of olive oil phenols in inflammation and cancer: molecular mechanisms and clinical implications. Current Medicinal Chemistry. 20: 4758-4776.

Carito, V., Venditti, A., Bianco, A., Ceccanti, M., Serrilli, A.M., Chaldakov, G., Tarani, L., De Nicolò, S., and Fiore, M. (2014). Effects of olive leaf polyphenols on male mouse brain NGF, BDNF and their receptors TrkA, TrkB and p75. Natural Product Research 28: 1970-1984.

Caruso, D., Visioli, F., Patelli, R., Galli, C., and Galli, G. (2001). Urinary excretion of olive oil phenols and their metabolites in humans. Metabolism 50: 1426-1428.

Cassano, A., Conidi, C., Giorno, L., and Drioli, E. (2013). Fractionation of olive mill wastewaters by membrane separation techniques. J. Hazard. Mater. 248-249: 185-193.

Castanas, E., Andricopoulos, N., Boskou, G., and Vercauteren, J. (2005). A Method for the Treatment of Olive Mill Waste Water, Patent WO 2005003037 (B1).

Castañer, O., Covas, M.-I., Khymenets, O., Nyyssonen, K., Konstantinidou, V., Zunft, H.-F., de la Torre, R., Muñoz-Aguayo, D., Vila, J., and Fitó, M. (2012). Protection of LDL from oxidation by olive oil polyphenols is associated with a downregulation of CD40-ligand expression and its downstream products in vivo in humans. Am. J. Clin. Nutr. 95: 1238-1244.

Cecchi, L., Migliorini, M., Zanoni, B., Breschi, C., and Mulinacci, N. (2018). An effective HPLC-based approach for the evaluation of the content of total phenolic compounds transferred from olives to virgin olive oil during the olive milling process. J. Sci. Food Agric. 98(10): 36363643.

Celano, R., Piccinelli, A.L., Pugliese, A., Carabetta, S., Di Sanzo, R., Rastrelli, L., and Russo, M. (2018). Insights into the Analysis of Phenolic Secoiridoids in Extra Virgin Olive Oil. J. Agric. Food Chem. 66: 6053-6063.

Charoenprasert, S., and Mitchell, A. (2012). Factors influencing fenolic compounds in table olives (Olea europea). J. Agric. Food Chem. 60 7081-7095.

Comandini, P., Lerma-Garcva, M.J., Massanova, P., Simo-Alfonso, E.F., and 
Toschia, T.G. (2015). Phenolic profiles of olive mill wastewaters treated by membrane filtration systems. Journal of Chemical Technology Biotechnology 90: 1086-1093.

Corona, G., Tzounis, X., Assunta Dessi, M., Deiana, M., Debnam, E.S., Visioli, F., and Spencer, J.P. (2006). The fate of olive oil polyphenols in the gastrointestinal tract: implications of gastric and colonic microfloradependent biotransformation. Free Radical Research 40: 647-658.

Cortesi, N., Azzolini, M., Rovelini, P., and Fedelii, E. (1995). Minor polar components of virgin olive oils: a hypothetical structure by LC-MS. Riv. Ital. Sost. Grasse 72: 241-251.

Covas, M.I., and Gaddi, A.V. (2011). Relationship of Olive Oil Polyphenols and Oxidation Biomarkers. J. Nutr.. Letters to the Editor 141: 2256.

Covas, M.-I., Nyyssönen, K., Poulsen, H.E., Kaikkonen, J., Zunft, H.-J.F., Kiesewetter, H., Gaddi, A., de la Torre, R., Mursu, J., Bäumler, H., Nascetti, S., Salonen, J.T., Fitó, M., Virtanen, J., and Marrugat, J. (2006) The effect of polyphenols in olive oil on heart disease risk factors: a randomized trial. Ann. Intern. Med. 145: 333-341.

Crawford, L.M., Holstege, D.M., and Wang, S.C. (2018). High-throughput extraction method for phenolic compounds in olive fruit (Olea europaea). J. Food Compost. Anal. 66: 136-144.

Cuomo, J., and Rabovskiy, A.B. (2001). Antioxidant Compositions Extracted from Olives and Olive By-products. Patent no. WO0145514 2001.

Dammak, M., Neves, A., Nabetanic, H., Isoda, H., Sayadi, S., and Nakajima, M. (2015). Transport properties of oleuropein through nanofiltration membranes. Food and Bioproducts Processing 94: 342-353.

De la Torre, R. (2008). Bioavailability of olive oil phenolic compounds in humans. Inflammopharmacology 16: 245-247.

De Martino, A., Arienzo, M., Iorio, M., Vinale, F., Lorito, M., Prenzler, P.D., Ryan, D., and Obied, H.K. (2011). Detoxification of olive mill wastewaters by zinc-aluminium layered double hydroxides. Applies Clay Science 53: 737-744.

de Torres, A., Espinola, F., Moya, M., Alcala, S., Vidal, A.M., and Castro, E. (2018). Assessment of phenolic compounds in virgin olive oil by response surface methodology with particular focus on flavonoids and lignans. Lwt-Food Science and Technology 90: 22-30.

Deiana, M. (2018). Function by extra virgin olive oil phenolic compounds. Food \& Function 9: 4085-4099.

Del Monaco, G., Officioso, A., D’Angelo, S., la Cara, F., Ionata, E., Marcolongo, L., Squillaci, G., Maurelli, L., and Morana, A. (2015). Characterization of extra virgin olive oils produced with typical Italian varieties by their phenolic profile. Food Chem. 184: 220-228.

Del Rio, D., Rodriguez-Mateos, A., Spencer, J.P.E., Tognolini, M., Borges, G., and Crozier, A. (2013). Dietary (poly)phenolics in human health: structures, bioavailability, and evidence of protective effects against chronic diseases. Antioxid. Redox Signal. 18: 1818-1892.

Deng, J.L., Xu, Z., Xiang, C.R., Liu, J., Zhou, L.J., Li, T., Yang, Z.S., and Ding, C.B. (2017). Comparative evaluation of maceration and ultrasonicassisted extraction of phenolic compounds from fresh olives. Ultrasonics Sonochemistry 37: 328-334.

Di Donna, L., Benabdelkamel, H., Mazzotti, F., Napoli, A., Nardi, M., and Sindona, G. (2011). High-throughput assay of oleopentanedialdheydes in extra virgin olive oil by the UHPLC-ESI-MS/MS and isotope dilution methods. Anal. Chem. 83: 1990-1995.

Domínguez-Perles, R., Auñón, D., Ferreres, F., and Gil-Izquierdo, A. (2017). Gender differences in plasma and urine metabolites from SpragueDawley rats after oral administration of normal and high doses of hydroxytyrosol, hydroxytyrosol acetate, and DOPAC. Eur. J. Nutr. 56(1): 215-224.

Edgecombe, S.C., Stretch, G.L., and Hayball, P.J. (2000). Oleuropein, an antioxidant polyphenol from olive oil, is poorly absorbed from isolated perfused rat intestine. J. Nutr. 130: 2996-3002.

EFSA Panel on Dietetic Products. (2011). Scientific Opinion on the substantiation of health claims related to polyphenols in olive and protection of LDL particles from oxidative damage (ID 1333, 1638, 1639, 1696 2865), maintenance of normal blood HDL cholesterol concentrations (ID 1639), mainte. EFSA Panel on Dietetic Products. EFSA Journal 9(4): 2033.

El-Abbassi, A., Kiai, H., and Hafidi, A. (2012). Phenolic profile and antioxidant activities of olive mill wastewater. Food Chem. 132: 406-412.

El-Abbassi, A., Kiai, H., Raiti, J., and Hafidi, A. (2014). Cloud point extrac- tion of phenolic compounds from pretreated olive mill wastewater. Journal of Environmental Chemical Engineering 2: 1480-1486.

Emmons, W., and Guttersen, C. (2005) Isolation of oleuropein aglycon from olive oil vegetation water. Patent US20050103711.

Ena, A., Pintucci, C., and Carlozzi, P. (2012). The recovery of polyphenols from olive mill waste using two adsorbing vegetable matrices. Journal of Biotechnology 157: 573-577.

Estruch, R., Ros, E., Salas-Salvadó, J., Covas, M.-I., Corella, D., Arós, F., Gómez-Gracia, E., Ruiz-Gutiérrez, V., Fiol, M., Lapetra, J., LamuelaRaventos, R.M., Serra-Majem, L., Pintó, X., Basora, J., Muñoz, M.A., Sorlí, J.V., Martínez, J.A., and Martínez-González, M.A. (2013). Primary Prevention of Cardiovascular Disease with a Mediterranean Diet. New England Journal of Medicine 368: 1279-1290.

Fabiani, R. (2016). Function. Food \& Function 7: 4145-4159.

Fanali, C., Della Posta, S., Vilmercati, A., Dugo, L., Russo, M., Petitti, T., Mondello, L., and de Gara, L. (2018). Extraction, Analysis, and Antioxidant Activity Evaluation of Phenolic Compounds in Different Italian Extra-Virgin Olive Oils. Molecules 23: 3249.

Farr, S.A., Price, T.O., Dominguez, L.J., Motisi, A., Saiano, F., Niehoff, M.L., Morley, J.E., Banks, W.A., Ercal, N., and Barbagallo, M. (2012). Extra virgin olive oil improves learning and memory in SAMP8 mice. Journal of Alzheimer's Disease 28: 81-92.

Fernandez-Bolanos, J., Rodriguez, G., and Rodriguez, R. (2002). Production in large quantities of highly purified hydroxytyrosol from liquid-solid waste of two phase olive oil processing or "alperujo". J. Agric. Food Chem. 50: 6804-6811.

Fernández-Poyatos, M.P., Ruiz-Medina, A., and Llorent-Martínez, E.J. (2019). Phytochemical profile, mineral content, and antioxidant ac tivity of Olea europaea L. cv. Cornezuelo table olives. Influence of in vitro simulated gastrointestinal digestion. Food Chem. 297: 124933.

Ferri, F., Bertin, L., Scoma, A., Marchetti, L., and Fava, F. (2011). Recovery of low molecular weight phenols through solid-phase extraction. Chemical Engineering J.166: 994-1001.

Firlbeck, D., Faulstich, M., Urmann, C.A., Azaizeh, H., Tafesh, A., and Riepl, H. (2013). Central composite design for optimal technology of concentrating vanillic acid using foam fractionation. Sep. Purif. Technol. 119: 28-34

Fitó, M., Cladellas, M., de la Torre, R., Martí, J., Muñoz, D., Schröder, H., Alcántara, M., Pujadas-Bastardes, M., Marrugat, J., López-Sabater, M.C., Bruguera, J., and Covas, M.I. (2008). Anti-inflammatory effect of virgin olive oil in stable coronary disease patients: a randomized, crossover controlled trial. European Journal of Clin. Nutr. 62: 570-574.

Francisco, V., Ruiz-Fernández, C., Lahera, V., Lago, F., Pino, J., Skaltsounis, L., González-Gay, M.A., Mobasheri, A., Gómez, R., Scotece, M., and Gualillo, O. (2019). Natural Molecules for Healthy Lifestyles: Oleocanthal from Extra Virgin Olive Oil. J. Agric. Food Chem. 67(14) 3845-3853.

Franco, M.N., Galeano-Díaz, T., López, O., Fernández-Bolaños, J.G., Sánchez, J., De Miguel, C., Gil, M.V., and Martín-Vertedor, D. (2014). Phenolic compounds and antioxidant capacity of virgin olive oil. Food Chem. 163: 289-298.

Frascari, D., Bacca, A.E.M., Zama, F., Bertin, L., Fava, F., and Pinelli, D. (2016). Olive mill wastewater valorisation through phenolic compounds adsorption in a continuous flow column. Chem. Eng. J. 283: 293-303.

Garcia-Castello, E., Cassano, A., Criscuoli, A., Conidi, C., and Drioli, E. (2010). Recovery and concentration of polyphenols from olive mil wastewaters by integrated membrane system. Water Res. 44: 38833892

Garcia-Serrano, P., Romero, C., Brenes, M., and Garcia-Garcia, P. (2019). Enrichment in phenolic compounds of black ripe olives through nano-filtration and vacuum evaporation techniques. Innovative Food Science \& Emerging Technologies 51: 73-79.

Garcia-Villalba, R., Carrasco-Pancorbo, A., Nevedomskaya, E., Mayboroda, O.A., Deelder, A.M., Segura- Carretero, A., and Fernandez-Gutierrez, A. (2010). Exploratory analysis of human urine by LC-ESI-TOF MS after high intake of olive oil: understanding the metabolism of polyphenols. Anal. Bioanal. Chem. 398: 463-475.

Gariboldi, P., Jommi, G., and Verotta, L. (1986). Secoiridoids from Olea Europaea. Phytochemistry 25(4): 865-896.

Genovese, A., Yang, N., Linforth, R., Sacchi, R., and Fisk, I. (2018). The role 
of phenolic compounds on olive oil aroma release. Food Research International 112: 319-327.

Ghanbari, R., Anwar, F., Alkharfy, K.M., Gilani, A.H., and Saari, N. (2012). Valuable nutrients and functional bioac- tives in different parts of olive (Olea europaea L.): a review. Int. J. Mol. Sci. 13: 3291-3340.

Gómez-Romero, M., García-Villalba, R., Carrasco-Pancorbo, A., and Fernández-Gutiérrez, A. (2012). Metabolism and bioavailability of olive oil polyphenols. In: Boskou, D. (Ed.). Olive Oil - Constituents, Quality, Health Properties and Bioconversions. Available from: https:// www.intechopen.com/books/olive-oil-constituents-quality-healthproperties-and-bioconversions/metabolism-and-bioavailability-ofolive-oil-polyphenols.

Gonzalez-Santiago, M., Fonolla, J., and Lopez-Huertas, E. (2010). Human absorption of a supplement containing purified hydroxytyrosol, a natural antioxidant from olive oil, and evidence for its transient association with low-density lipoproteins. Pharmacol. Res. 61: 364-370.

Gorzynik-Debicka, M., Przychodzen, P., Cappello, F., Kuban-Jankowska, A. Gammazza, A.M., Knap, N., Wozniak, M., and Gorska-Ponikowska, M. (2018). Potential Health Benefits of Olive Oil and Plant Polyphenols. Int. J. Mol. Sci. 19: 1-13.

Gotsis, E., Anagnostis, P., Mariolis, A., Vlachou, A., Katsiki, N., and Karagiannis, A. (2015). Health benefits of the Mediterranean Diet: an update of research over the last 5 years. Angiology 66: 304-318.

Goulas, V., Charisiadis, P., Gerothanassis, I.P., and Manganaris, G.A. (2012). Classification, biotransformation and antioxidant activity of olive fruit biophenols: a review. Current Bioactive Compounds 8: 232-239.

Grosso, G., Buscemi, S., Galvano, F., Mistretta, A., Marventano, S., La Vela, V., Drago, F., Gangi, S., Basile, F., and Biondi, A. (2013). Mediterranean diet and cancer: epidemiological evidence and mechanism of selected aspects. BMC Surgery 13(Suppl. 2): S14.

Guasch-Ferré, M., Hruby, A., Salas-Salvadó, J., Martínez-González, M.A., Sun, Q., Willett, W.C., and Hu, F.B. (2015). Olive oil consumption and risk of type 2 diabetes in US women. Am. J. Clin. Nutr. 102(2): 479-486.

Guasch-Ferré, M., Hu, F.B., Martínez-González, M.A., Fitó, M., Bulló, M. Estruch, R., Ros, E., Corella, D., Recondo, J., Gómez-Gracia, E., Fiol, M., Lapetra, J., Serra-Majem, L., Muñoz, M.A., Pintó, X., LamuelaRaventós, R.M., Basora, J., Buil-Cosiales, P., Sorlí, J.V., Ruiz-Gutiérrez, V., Martínez, J.A., and Salas-Salvadó, J. (2014). Olive oil intake and risk of cardiovascular disease and mortality in the PREDIMED Study. BMC Medicine 12: 78 .

Guo, X., Tresserra-Rimbau, A., Estruch, R., Martinez-Gonzalez, M.A., Medina-Remon, A., Fitó, M., Corella, D., Salas-Salvadó, J., Portillo, M.P., Moreno, J.J., and et al (2017). Polyphenol levels are inversely correlated with body weight and obesity in an elderly population after 5 years of follow up (The Randomised PREDIMED Study). Nutrients 9: 452.

Gutfinger, T. (1981). Polyphenols in olive oils, J. Amer. Oil Chem. Soci. 58: 966-968.

Hernáez, Á., Fernández-Castillejo, S., Farràs, M., Catalán, Ú., Subirana, I., Montes, R., Solà, R., Muñoz-Aguayo, D., Gelabert-Gorgues, A., DíazGil, Ó., Nyyssönen, K., Zunft, H.-J.F., de la Torre, R., Martín-Peláez, S. Pedret, A., Remaley, A.T., Covas, M.-I., and Fitó, M. (2014). Olive oi polyphenols enhance high-density lipoprotein function in humans: a randomized controlled trial. Arteriosclerosis, Thrombosis, and Vascular Biology 34: 2115-2119.

Inouye, H., and Uesato, S. (1986). Biosynthesis of Iridoids and Secoiridoids. In: Herz, W., Grisebach, H., Kirby, G.W., and Tamm, Ch. (Ed.). Progress in the Chemistry of Organic Natural Products. Springer, Verlag, Wien New York, pp. 169-220.

International Olive Council (IOC) testing methods. (2009). Determination of biophenols in olive oil by HPLC. COI/T.20/Doc No 29, November.

Jerman Klen, T., and Mozetič-Vodopivec, B. (2012). Optimisation of olive oil phenol extraction conditions using a high-power probe ultrasonication. Food Chem. 134: 2481-2488.

Jerman-Klen, T., Golc-Wondra, A., Vrhovšek, U., and Mozetič-Vodopivec, B. (2015). Phenolic Profiling of Olives and Olive Oil Process-Derived Matrices Using UPLC-DAD-ESI-QTOF-HRMS Analysis. J. Agric. Food Chem. 63: 3859-3872.

Kailis, S.G., and Kiritsakis, A. (2017). Table Olives, Processing, Nutritional and Health Implications. In: Kiritsakis, A.P., and Shahidi, F. (Ed.). Ol- ives and Olive oil as Functional Foods: Bioactivity, Chemistry and Processing. Wiley, Co, pp. 295-324.

Kamiloglu, S., Demirci, M., Selen, S., Toydemir, G., Boyacioglu, D., and Capanoglu, E. (2014). Home processing of tomatoes (Solanum lycopersicum): effects on in vitro bioaccessibility of total lycopene, phenolics, flavonoids, and antioxidant capacity. J. Sci. Food Agric. 94: 2225-2233.

Kanakis, P., Termentzi, A., Michel, T., Gikas, E., Halabalaki, M., and Skaltsounis, A.L. (2013). From olive drupes to olive oil. An HPLC-orbitrapbased qualitative and quantitative exploration of olive key metabolites. Planta Medica 79: 1576-1587.

Kano, S., Komada, H., Yonekura, L., Sato, A., Nishiwaki, H., and Tamura, H. (2016). Absorption, metabolism, and excretion by freely moving rats of 3, 4-DHPEA-EDA and related polyphenols from olive fruits (Olea europaea). Journal of Nutrition and Metabolism 2016: 9104208

Karkoula, E., Skantzari, A., Melliou, E., and Magiatis, P. (2014). Quantitative Measurement of Major Secoiridoid Derivatives in Olive Oil Using qNMR. Proof of the Artificial Formation of Aldehydic Oleuropein and Ligstroside Aglycon Isomers. J. Agric. Food Chem. 62: 600-607.

Keceli, T.M., Kamiloglu, S., and Capanoglu, E. (2017). Phenolic Compounds of Olives and Olive Oil and their Bioavailability. In: Kiritsakis, A.P., and Shahidi, F. (Ed.). Olives and Olive oil as Functional Foods: Bioactivity, Chemistry and Processing. Wiley, Co, pp. 457-471.

Khalatbary, A.R. (2013). Olive oil phenols and neuroprotection. Nutr. Neurosci. 16: 243-249.

Khera, A.V., Cuchel, M., de la Llera-Moya, M., Rodrigues, A., Burke, M.F. Jafri, K., French, B.C., Phillips, J.A., Mucksavage, M.L., Wilensky, R.L., Mohler, E.R., Rothblat, G.H., and Rader, D.J. (2011). Cholesterol efflux capacity, high-density lipoprotein function, and atherosclerosis. N Engl J. Med. 364: 127-135.

Khymenets, O., Crespo, M.C., Dangles, O., Rakotomanomana, N., AndresLacueva, C., and Visioli, F. (2016). Human hydroxytyrosol's absorption and excretion from a nutraceutical. J. Funct. Foods 23: 278-282.

Khymenets, O., Farre, M., Pujadas, M., Ortiz, E., Joglar, J., Covas, M.I., and De la Torre, R. (2011). Direct analysis of glucuronidated metabolites of main olive oil phenols in human urine after dietary consumption of virgin olive oil. Food Chem. 126: 306-314.

Kiralan, M., Ozkan, G., Koyluoglu, F., Ugurl, H.A., and Kiritsakis, A. Effect of cultivation area and climatic conditions on volatiles of virgin olive oil. Eur. J. Lipid Sci. Technol. 114(5): 552-557.

Kiritsakis, A., and Shahidi, F. (2017). Olive Oil Quality and its Relation to Functional Bioactives and their Properties. In: Kiritsakis, A.P., and Shahidi, F. (Ed.). Olives and Olive oil as Functional Foods: Bioactivity, Chemistry and Processing. Wiley, Co, pp. 205-219.

Kiritsakis, K., Goula, A.M., Adamopoulos, K.G., and Gerasopoulos, D. (2017a). Valorization of Olive Leaves: Spray Drying of Olive Leaf Extract. Waste and Biomass Valorization 9: 619-633.

Kiritsakis, K., Melliou, E., Magiatis, P., and Gerasopoulos, D. (2017b). Enhancement of Bioactive Phenols and Quality Values of Olive Oil by Recycling Olive Mill Waste Water. J Am Oil Chem Soc. 94(8): 10771085

Kiritsakis, A., Keceli, M.T., and Kiritsakis, K. (2020). Olive Oil. In: Shahidi, F. (Ed.). Bailey's Industrial Oil and Fat Products, $6^{\text {th }}$. Wiley, Co, .

Kiritsakis, A., and Markakis, P. (1987). Olive Oil-A Review. Adv. Food Res. 31: 453-482.

Kiritsakis, A., and Shahidi, E. (2017). Olive Oil Quality and its Relation to the Functional Bioactives and their Properties. In: Kiritsakis, A.P., and Shahidi, F. (Ed.). Olives and Olive oil as Functional Foods: Bioactivity, Chemistry and Processing. Wiley, Co, pp. 205-219.

Kiritsakis, A.K. (1998). Olive oil: from the tree to the table, 2 nd ed. Food \& Nutrition Press, Trumbull, CT, USA,

Kiritsakis, K., Kontominas, M.G., Kontogiorgis, C., Hadjipavlou-Litina, D. Moustakas, A., and Kiritsakis, A. (2010). Composition and antioxidant activity of olive leaf extracts from Greek olive cultivars. Journal of the American Oil Chemists' Society 87: 369-376.

Kiritsakis, K., Rodriguez-Perez, C., Gerasopoulos, D., and Segura- Carretero, A. (2017c). Olive oil enrichment in phenolic compounds during malaxation in the presence of olive leaves or olive mill wastewater extracts. Eur. J. Lipid Sci. Technol. 119: 1600425.

Klen, T.J., and Vodopivec, B.M. (2012). Optimisation of Olive Oil Phenol Extraction Conditions Using a High-Power Probe Ultrasonication. J. 
Food Chem. 134(4): 2481-2488.

Knorr, D., Zenker, M., Heinz, V., and Lee, D.U. (2004). Applications and potential of ultrasonics in food processing. Trends in Food Science and Technology 15: 261-266.

Kontush, A., and Chapman, M.J. (2006). Functionally defective high-density lipoprotein: a new therapeutic target at the crossroads of dyslipidemia, inflammation, and atherosclerosis. Pharmacol Rev. 58: 342-374.

Kotronoulas, A., Pizarro, N., Serra, A., Robledo, P., Joglar, J., Rubio, L., Hernaez, A., Tormos, C., Motilva, M.J., Fito, M., Covas, M.I., Sola, R., Farre, M., Saez, G., and De la Torre, R. (2013). Dose-dependent metabolic dispo- sition of hydroxytyrosol and formation of mercapturates in rats. Pharmacol. Res. 77: 47-56.

Kountouri, A.M., Mylona, A., Kaliora, A.C., and Andrikopoulos, N.K. (2007). Bioavailability of the phenolic com- pounds of the fruits (drupes) of Olea europaea (olives): impact on plasma antioxidant status in humans. Phytomedicine 14: 659-667.

Lafka, T-I., Lazou, A.E., Sinanoglou, V.J., and Lazos, E.S. (2011). Phenolic and antioxidant potential of olive oil mill wastes. Food Chem. 125: 92-98.

Lam, C.K., Zhang, Z., Yu, H., Tsang, S.Y., Huang, Y., and Chen, Z.Y. (2008). Apple polyphenols inhibit plasma CETP activity and reduce the ratio of non-HDL to HDL cholesterol. Mol. Nutr. Food Res. 52: 950-958.

Liberatore, L., Procida, G., D'Alessandro, A., and Cichelli, A. Solid-phase extraction and gas chromatographic analysis of phenolic compounds in virgin olive oil. Food Chem. 73: 119-124.

Liu, D., and Wang, M. (2008). Novel use of hydroxytyrosol and olive extracts/concentrates containing in it. Patent US0130620 A1.

Lodolini, E.M., Cabrera-Banegil, M., Fernandez, A., Delgado-Adamez, J., Ramirez, R., and Martin-Vertedor, D. (2019). Monitoring of acrylamide and phenolic compounds in table olive after high hydrostatic pressure and cooking treatments. Food Chem. 286: 250-259.

López de las Hazas, M.C., Piñol, C., Macià, A., Romero, M.P., Pedret, A., Solà, R., Rubió, L., and Motilva, M.J. (2016). Differential absorption and metabolism of hydroxytyrosol and its precursors oleuropein and secoiridoids. J. Funct. Foods 22: 52-63.

Loued, S., Berrougui, H., Componova, P., Ikhlef, S., Helal, O., and Khalil, A. (2013). Extra-virgin olive oil consumption reduces the age-related decrease in HDL and paraoxonase 1 anti-inflammatory activities. The Br. J. Nutr. 110: 1272-1284.

Lozano-Castellón, J., López-Yerena, A., Rinaldi de Alvarenga, J.F., CastilloAlba, J.R., Vallverdú-Queralt, A., Escribano-Ferrer, E., and LamuelaRaventós, R.M. (2019). Health-promoting properties of oleocanthal and oleacein: Two secoiridoids from extra-virgin olive oil. Crit. Rev. Food Sci. Nutr. 0: 1-17.

Lozano-Sanchez, J., Giambanelli, E., Quirantes-Pine, R., Cerretani, L., Bendini, A., Segura-Carretero, A., and Fernandez-Gutierrez, A. (2011). Wastes generated during the storage of extra virgin olive oil as a natural source of phenolic compounds. J. Agric. Food Chem. 59: 11491-11500.

Luigi Iorio, E. (2020). Personal communication.

Magiatis, P., and Melliou, E. (2015). Method of colorimetric determination of oleocanthal and oleacein in olive oil - treatment and measurement kit. GR1008569 (B) 2015-09-04.

Manach, C., Scalbert, A., Morand, C., Rémésy, C., and Jiménez, L. (2004). Polyphenols: food sources and bioavailability. Am. J. Clin. Nutr. 79: 727-747.

Manna, C., Galletti, P., Maisto, G., Cucciolla, V., D’Angelo, S., and Zappia, V. (2009). Transport mechanism and metabolism of olive oil hydroxytyrosol in Caco-2 cells. FEBS letters 470: 341-344

Marranzano, M., Ray, S., Godos, J., and Galvano, F. (2018). Association between dietary flavonoids intake and obesity in a cohort of adults living in the Mediterranean area. Int. J. Food Sci. Nutr. 26: 1-10.

Martínez-González, M.Á., Toledo, E., Arós, F., Fiol, M., Corella, D., SalasSalvadó, J., Ros, E., Covas, M.I., Fernández-Crehuet, J., Lapetra, J., Muñoz, M.A., Fitó, M., Serra-Majem, L., Pintó, X., Lamuela-Raventós, R.M., Sorlí, J.V., Babio, N., Buil- Cosiales, P., Ruiz-Gutierrez, V., Estruch, R., and Alonso, A. (2014). Extra virgin olive oil consumption reduces risk of atrial fibrillation: the PREDIMED (Prevención con Dieta Mediterránea) trial. Circulation 130: 18-26.

Martínez-Lapiscina, E.H., Clavero, P., Toledo, E., San Julián, B., SanchezTainta, A., Corella, D., Lamuela-Raventós, R.M., Martínez, J.A., and
Martínez-Gonzalez, M.Á. (2013). Virgin olive oil supplementation and long-term cognition: the PREDIMED-NAVARRA randomized, trial. The Journal of Nutrition, Health and Aging 17: 544-552.

Martín-Peláez, S., Covas, M.I., Fitó, M., Kušar, A., and Pravst, I. (2013). Health effects of olive oil polyphenols: recent advances and possibilities for the use of health claims. Mol. Nutr. Food Res. 57: 760-771.

Melliou, E., Zweigenbaum, J.A., and Mitchell, A.E. (2015). Ultrahigh-pressure liquid chromatography triple-quadrupole tandem mass spectrometry quantitation of polyphenols and secoiridoids in californiastyle black ripe olives and dry salt-cured olives. J. Agric. Food Chem. 63: 2400-2405.

Melliou, E., Diamantakos, P., and Magiatis, P. (2017). New Analytical Trends for the Measurement of Phenolic Substances of Olive Oil and Olives with Significant Biological and Functional Importance Related to Health Claims. In: Kiritsakis, A.P., and Shahidi, F. (Ed.). Olives and Olive oil as Functional Foods: Bioactivity, Chemistry and Processing. Wiley, Co, pp. 569-582.

Mettouchi, S., Sacchi, R., Moussa, Z.E.D.O., Paduano, A., Savarese, M., and Tamendjari, A. (2016). Effect of Spanish style processing on the phenolic compounds and antioxidant activity of Algerian green table olives. Grasas Y Aceites 67(1): 1-11.

Miro-Casas, E., Albadalejo, M.F., Planells, M.I.C., Colomer, M.F., Raventos, R.M.L., and De la Torre, R. (2001b). Tyrosol bioavailability in humans after ingestion of virgin olive oil. Clin. Chem. 47: 341-343.

Miro-Casas, E., Albaladejo, M.F., Covas, M.I., Rodriguez, J.O., Colomer, E.M., Raventos, R.M.L., and De la Torre, R. (2001a). Capillary gas chromatography-mass spectrometry quantitative determination of hydroxytyrosol and tyrosol in human urine after olive oil intake. Anal. Biochem. 294: 63-72.

Miro-Casas, E., Covas, M.I., Farre, M., Fito, M., Ortuno, J., Weinbrenner, T., Roset, P., and de la Torre, R. (2003). Hydroxytyrosol disposition in humans. Clin. Chem. 49: 945-952.

Monasterio, R.P., Olmo-García, L., Bajoub, A., Fernández-Gutiérrez, A., and Carrasco-Pancorbo, A. (2017). Phenolic Compounds Profiling of Virgin Olive Oils from Different Varieties Cultivated in Mendoza, Argentina, by Using Liquid Chromatography-Mass Spectrometry. J. Agric. Food Chem. 65(37): 8184-8195.

Montano, A., Hernandez, M., Garrido, I., Llerena, J.L., and Espinosa, F. (2016). Fatty Acid and Phenolic Compound Concentrations in Eight Different Monovarietal Virgin Olive Oils from Extremadura and the Relationship with Oxidative Stability. Int. J. Mol. Sci. 17(11): 1960.

Montedoro, G.F., Servilli, M., Bardioli, M., and Miniati, E. (1992). Simple and hydrolyzable phenolic compounds in virgin olive oil 2. Initial characterization of the hydrolyzable fraction. J. Agric. Food Chem. 40: 1571-1576.

Mosele, J.I., Martin-Pelaez, S., Macia, A., Farras, M., Valls, R.M., Catalan, U., and Motilva, M.J. (2014). Faecal microbial metabolism of olive oil phenolic compounds: In vitro and in vivo approaches. Mol. Nutr Food Res. 58: 1809-1819.

Neveu, V., Perez-Jiménez, J., Vos, F., Crespy, V., du Chaffaut, L., Mennen, L., Knox, C., Eisner, R., Cruz, J., Wishart, D., and Scalbert, A. (2010). Phenol-Explorer: an online comprehensive database on polyphenol contents in foods. Database (Oxford) 2010: bap024.

Nunes, A.V.M.M., Crespo, J.P.S.G., Santos, J.C.D., Matias, A.F., and Duarte, C.M. (2007). Method of obtaining a natural hydroxytyrosolrich concentrate from olive tree residues and subproducts using clean technologies. United States Patent Appl. Publ. within the TVPP US20080020960.

Ochando-Pulido, J.M., Victor-Ortega, M.D., and Martinez-Ferez, A. (2015). On the cleaning procedure of a hydrophilic reverse osmosis membrane fouled by secondary-treated olive mill wastewater. Chem. Eng. J. 260: 142-151.

Oi-Kano, Y., Iwasaki, Y., Toshiyuki, N., Watanabe, T., Goto, T., Kawada, T., Watanabe, K., and Iwai, K. (2017). Oleuropein aglycone enhances UCP1 expression in brown adipose tissue in high-fat-diet-induced obese rats by activating -adrenergic signaling. J. Nutr. Biochem. 40 : 209-218.

Oliveras-Lopez, M.J., Berna, G., Jurado-Ruiz, E., De la Serrana, H.L.G., and Martin, F. (2014). Consumption of extra-virgin olive oil rich in phenolic compounds has beneficial antioxidant effects in healthy human adults. J. Funct. Foods 10: 475-484.

Olivero-David, R., Mena, C., Pérez-Jimenez, M.A., Sastre, B., Bastida, S., 
Márquez-Ruiz, G., and Sánchez-Muniz, F.J. (2014). Influence of Picual olive ripening on virgin olive oil alteration and stability during potato frying. J. Agric. Food Chem. 62: 11637-11646.

Otvos, J.D., Collins, D., Freedman, D.S., Shalaurova, I., Schaefer, E.J., McNamara, J.R., Bloomfield, H.E., and Robins, S.J. (2006). Low-density lipoprotein and high-density lipoprotein particle subclasses predict coronary events and are favorably changed by gemfibrozil therapy in the Veterans Affairs High-Density Lipoprotein Intervention Trial. Circulation 113: 1556-1563.

Owen, R.W., Haubner, R., Würtele, G., Hull, W.E., Spiegelhalder, B., and Bartsch, H. (2004). Olives and olive oil in cancer prevention. Eur. J. Cancer Prev. 13: 319-326.

Paiva-Martins, F., Fernandes, J., Rocha, S., Nascimento, H., Vitorino, R., Amado, F., Borges, F., Belo, L., and Silva, A.S. (2009). Effects of olive oil polyphenols on erythrocyte oxidative damage. Mol Nutr Food Res 53: 609-616.

Paraskeva, C.A., Papadakis, V.G., Tsarouchi, E., Kanellopoulou, D.G., and Koutsoukos, P.G. (2007). Membrane processing for olive mill wastewater fractionation. Desalination 213: 218-229.

Pastor, A., Rodríguez-Morató, J., Olesti, E., Pujadas, M., Pérez-Mañá, C., Khymenets, O., Fitó, M., Covas, M.I., Solá, R., Motilva, M.J., Farré, M., and la Torre, R. (2016). Analysis of free hydroxytyrosol in human plasma following the administration of olive oil. Journal of Chromatography A 1437: 183-190.

Pedan, V., Popp, M., Rohn, S., Nyfeler, M., and Bongartz, A. (2019). Characterization of Phenolic Compounds and Their Contribution to Sensory Properties of Olive Oil. Molecules 24(11): 2041.

Peres, F., Martins, L.L., Mourato, M., Vitorino, C., Antunes, P., and FerreiraDias, S. (2016). Phenolic compounds of 'Galega Vulgar' and 'Cobrancosa' olive oils along early ripening stages. Food Chem. 211: 51-58.

Phenol-Explorer. Database on polyphenol content. http://www.phenolexplorer.eu, Accessed 18 Nov 2019.

Pinto, J., Paiva-Martins, F., Corona, G., Debnam, E.S., Jose Oruna-Concha M., Vauzour, D., Gordon, M.H., and Spencer, J.P. (2011). Absorption and metabolism of olive oil secoiridoids in the small intestine. Br. J. Nutr. 105: 1607-1618.

Pizarro, M.L., Becerra, M., Sayago, A., Beltrán, M., and Beltrán, R. (2012). Comparison of Different Extraction Methods to Determine Phenolic Compounds in Virgin Olive Oil. Food Analytical Methods 6: 123-132.

Pizzichini, M., and Russo, C. (2005). Process for Recovering the Components of Olive Mill Wastewater with Membrane Technologies. Patent WO2005123603.

Psaltopoulou, T., Kosti, R.I., Haidopoulos, D., Dimopoulos, M., and Panagiotakos, D.B. (2011). Olive oil intake is inversely related to cancer prevalence: a systematic review and a meta-analysis of 13,800 patients and 23,340 controls in 19 observational studies. Lipids in Health and Disease. 10: 127.

Quintero-Flórez, A., Pereira-Caro, G., Sánchez-Quezada, C., Moreno-Rojas, J.M., Gaforio, J.J., Jimenez, A., and Beltrán, G. (2018). Effect of olive cultivar on bioaccessibility and antioxidant activity of phenolic fraction of virgin olive oil. Eur. J. Nutr. 57(5): 1925-1946.

Ramos-Escudero, F., Morales, M.T., and Asuero, A.G. (2015). Characterization of bioactive compounds from mono- varietal virgin olive oils: relationship between phenolic compounds-antioxidant capacities. International Journal of Food Properties 18: 348-358.

Rinaldi de Alvarenga, J.F., Quifer-Rada, P., Juliano, F.F., Hurtado-Barroso, S., Torrado-Prat, X., and Lamuela-Raventós, R.M. (2019). Using Extra Virgin Olive Oil to Cook Vegetables Enhances Polyphenol and Carotenoid Extractability: A Study Applying the sofrito Technique. Molecules 24: 1-17.

Rios, J.J., Gil, M.J., and Gutierrez-Rosales, F. (2005). Solid-phase extraction gas chromatography-ion trap-mass spectrometry qualitative method for evaluation of phenolic compounds in virgin olive oil and structural confirmation of oleuropein and ligstroside aglycons and their oxidation products. Journal of Chromatography A 1093: 167-176.

Rodrigues, N., Casal, S., Pinho, T., Peres, A.M., Bento, A., Baptista, P., and Pereira, J.A. (2019). Ancient olive trees as a source of olive oils rich in phenolic compounds. Food Chem. 276: 231-239.

Romani, A., leri, F., Urciuoli, S., Noce, A., Marrone, G., Nediani, C., and Bernini, R. (2019). Health Effects of Phenolic Compounds Found in Extra-Virgin Olive Oil, By-Products, and Leaf of Olea europaea L. Nu- trients 11(8): 1776

Ros, E., Martínez-González, M.A., Estruch, R., Salas-Salvadó, J., Fitó, M. Martínez, J.A., and Corella, D. (2014). Mediterranean diet and cardiovascular health: teachings of the PREDIMED study. Adv. Nutr. 5: 330S-336S.

Rosello-Soto, E., Koubaa, M., Moubarik, A., Lopes, R.P., Saraiva, J.A., Boussetta, N., Grimi, N., and Barba, F.J. (2015). Emerging opportunities for the effective valorization of wastes and by-products generated during olive oil production process: nonconventional methods for the recovery of high-added value compounds. Trends in Food Science \& Technology 45: 296-310.

Rubio, L., Macia, A., Valls, R.M., Pedret, A., Romero, M.P., Sola, R., and Motilva, M.J. (2012a). A new hydroxyty- rosol metabolite identified in human plasma: hydroxytyrosol acetate sulphate. Food Chem. 134: 1132-1136.

Rubio, L., Valls, R.M., Macia, A., Pedret, A., Giralt, M., Romero, M.P., De la Torre, R., Covas, M.I., Sola, R., and Motilva, M.J. (2012b). Impact of olive oil phenolic concentration on human plasmatic phenolic metabolites. Food Chem. 135: 2922-2929.

Russo, C. (2007). A new membrane process for the selective fractionation and total recovery of polyphenols, water and organic substances from vegetation waters (VW). J. Membr. Sci. 288: 239-246.

Sabbah, I., Marsook, T., and Basheer, S. (2004). The effect of pretreatment on anaerobic activity of olive mill wastewater using batch and continuous systems. Process Biochemistry 39: 1947-1951.

Sahin, S., and Samli, R. (2013). Optimization of olive leaf extract obtained by ultrasound-assisted extraction with response surface methodology. Ultrasonics Sonochemistry 20: 595-602.

Saitta, M., Lo Curto, S., Salvo, F., Di Bella, G., and Dugo, G. (2002). Gas chromatographic-tandem mass spectrometric identification of phenolic compounds in Sicilian olive oils. Anal. Chim. Acta 466: 335-344.

Salvini, S., Sera, F., Caruso, D., Giovannelli, L., Visioli, F., Saieva, C., Masala, G., Ceroti, M., Giovacchini, V., Pitozzi, V., Galli, C., Romani, A., Mulinacci, N., Bortolomeazzi, R., Dolara, P., and Palli, D. (2006). Daily consumption of a high-phenol extra-virgin olive oil reduces oxidative DNA damage in postmenopausal women. Br. J. Nutr. 95: 742-751.

Samieri, C., Féart, C., Proust-Lima, C., Peuchant, E., Tzourio, C., Stapf, C., Berr, C., and Barberger-Gateau, P. (2011). Olive oil consumption, plasma oleic acid, and stroke incidence: the Three-City Study. Neurology 77: 418-425.

Santangelo, C., Filesi, R., Vari, R., Scazzocchio, B., Filardi, T., Fogliano, V., D’Archivio, M., Giovannini, C., Lenzi, A., Morano, S., and Masella, R (2016). Consumption of extra-virgin olive oil rich in phenolic compounds improves metabolic control in patients with type 2 diabetes mellitus: a possible involvement of reduced levels of circulating visfatin. J. Endocrinol. Invest. 39: 1295-1301.

Santi, C.A., Cortes, S., D'Acqui, L.P., Sparvoli, E., and Pushparaj, B. (2008) Reduction of organic pollutants in olive mill wastewater by using different mineral substrates as adsorbents. Bioresource Technology 99: 1945-1951.

Sarbishegi, M., Mehraein, F., and Soleimani, M. (2014). Antioxidant role of oleuropein on midbrain and dopaminergic neurons of substantia nigra in aged rats. Iran Biomed. J. 18: 16-22.

Scoma, A., Bertin, L., Zanaroli, G., Fraraccio, S., and Fava, F. (2011). A physicochemical-biotechnological approach for an integrated valorization of olive mill wastewater. Bioresource Technology 102: 10273-10279.

Segura-Carretero, A., Carrasco-Pancorbo, A., Bendini, A., Cerretani, L., and Fernández-Gutiérrez, A. (2010). Chapter 56 - Analytical Determination of Polyphenols in Olive Oil. In: Preedy, V.R., and Watson, R.R (Ed.). Olives and Olive Oil in Health and Disease Prevention. Academic Press, San Diego, pp. 509-523.

Serra, A., Rubio, L., Borras, X., Macia, A., Romero, M.P., and Motilva, M.J. (2012). Distribution of olive oil phenolic compounds in rat tissues af ter administration of a phenolic extract from olive cake. Mol. Nutr Food Res. 56: 486-496.

Serreli, G., and Deiana, M. (2018). Biological relevance of extra virgin olive oil polyphenols metabolites. Antioxidants 7: 11-13.

Servili, M., Esposto, S., Veneziani, G., Urbani, S., Taticchi, A., Di Maio, I., Selvaggini, R., Sordini, B., and Montedoro, G. (2011). Improvement of bioactive phenol content in virgin olive oil with an olive-vegetation water concentrate produced by membrane treatment. Food Chem. 


\section{4: 1308-1315.}

Silva, S., Garcia-Aloy, M., Figueira, M.E., Combet, E., Mullen, W., and Bronze, M.R. (2018). High resolution mass spectrometric analysis of secoiridoids and metabolites as biomarkers of acute olive oil intakeAn approach to study interindividual variability in humans. Mol. Nutr. Food Res. 62(2): 1700065

Sinesio, F., Moneta, E., Raffo, A., Lucchetti, S., Peparaio, M., D’Aloise, A. and Pastore, G. (2015). Effect of extraction conditions and storage time on the sensory profile of monovarietal extra virgin olive oil (cv Carboncella) and chemical drivers of sensory changes. LWT - Food Science and Technology 63: 281-288.

Singh, C.K., Sahu, J.N., Mahalik, K.K., Mohanty, C.R., Raj Mohan, B., and Meikap, B.C. (2008). Studies on the removal of $\mathrm{Pb}(\mathrm{II})$ from wastewater by activated carbon developed from Tamarind wood activated with sulphuric acid. J. Hazard. Mater. 153: 221-228.

Soler, A., Romero, M.P., Macià, A., Saha, S., Furniss, C.S.M., Kroon, P.A., and Motilva, M.J. (2010). Digestion stability and evaluation of the metabolism and transport of olive oil phenols in the human smallintestinal epithelial Caco-2/TC7 cell line. Food Chem. 119: 703-714.

Stasinakis, A.S., Elia, I., Petalas, A.V., and Halvadakis, C.P. (2008). Removal of total phenols from olive-mill wastewater using an agricultural byproduct, olive pomace. J. Hazard. Mater. 160: 408-413.

Stoller, M., and Chianese, A. (2006). Optimization of membrane batch processes by means of the critical flux theory. Desalination 191: 62-70.

Suarez, M., Valls, R.M., Romero, M.P., Macia, A., Fernandez, S., Giralt, M., Sola, R., and Motilva, M.J. (2011). Bioavailability of phenols from a phenol-enriched olive oil. Br. J. Nutr. 106: 1691-1701.

Syvänne, M., Ahola, M., Lahdenperäm, S., Kahri, J., Kuusi, T., Virtanen, K.S., and Taskinen, M.R. (1995). High density lipoprotein subfractions in non-insulin-dependent diabetes mellitus and coronary artery disease. J. Lipid Res. 36: 573-582.

Takaç, S., and Karakaya, A. (2009). Recovery of phenolic antioxidants from olive mill wastewater. Recent Patents in Chemical Engineering 2: 230-237.

Tang, G., Huang, Y., Zhang, T., Wang, Q., Crommen, J., Fillet, M., and Jiang, Z. (2018). Determination of phenolic acids in extra virgin olive oil using supercritical fluid chromatography coupled with single quadrupole mass spectrometry. Journal of Pharmaceutical and Biomedical Analysis 157: 217-225.

Tasioula-Margari, M., and Okogeri, O. (2001). Simultaneous determination of phenolic compounds and tocopherols in virgin olive oil using HPLC and UV detection. Food Chem. 74: 377-383.

Tornberg, E., and Galanakis, C.M. (2008). Olive Waste Recovery, World Intellectual Property Organization, WO/2008/082343.

Tresserra-Rimbau, A., and Lamuela-Raventós, R.M. (2017). Olives and Olive Oil: A Mediterranean Source of Polyphenols. In: Kiritsakis, A.P., and Shahidi, F. (Ed.). Olives and Olive oil as Functional Foods: Bioactivity, Chemistry and Processing. Wiley, Co, pp. 457-471.

Tsimidou, M., Papadopoulos, G., and Boskou, D. (1992). Determination of phenolic compounds in virgin olive oil by reversed-phase HPLC with emphasis on UV detection. Food Chem. 44: 53-60.

Tsimidou, M.Z., Nenadis, N., Servili, M., García-González, D.L., and Toschi, T.G. (2018). Why Tyrosol Derivatives Have to Be Quantified in the Calculation of "Olive Oil Polyphenols". Content to Support the Health Claim Provisioned in the EC Reg. 432/2012. Eur. J. Lipid Science Technology 120: 1800098.

Tuck, K.L., Freeman, M.P., Hayball, P.J., Stretch, G.L., and Stupans, I. (2001). The in vivo fate of hydroxytyrosol and tyrosol, antioxidant phenolic constituents of olive oil, after intravenous and oral dosing of labeled compounds to rats. J. Nutr. 131: 1993-1996.

Tuck, K.L., Hayball, P.J., and Stupans, I. (2002). Structural characterization of the metabolites of hydroxytyrosol, the principal phenolic component in olive oil, in rats. J. Agric. Food Chem. 50: 2404-2409.

Turano, E., Curcio, S., De Paola, M.G., Calabro, V., and Iorio, G. (2002). An integrated centrifugation ultrafiltration system in the treatment of olive mill wastewater. J. Membr. Sci. 202: 519-531.

Valko, M., Leibfritz, D., Moncol, J., Cronin, M.T.D., Mazur, M., and Telser, J. (2007). Free radicals and antioxidants in normal physiological functions and human disease. The International Journal of Biochemistry \& Cell Biology 39: 44-84.

Venturi, F., Sanmartin, C., Taglieri, I., Nari, A., Andrich, G., Terzuoli, E., Don- nini, S., Nicolella, C., and Zinnai, A. (2017). Development of PhenolEnriched Olive Oil with Phenolic Compounds Extracted from Wastewater Produced by Physical Refining. Nutrients 9(8): 916

Villanova, L., Fasiello, G., and Merendino, A. (2008). Process for the Recovery of Tyrosol and Hydroxytyrosol from Oil Mill Wastewaters and Catalytic Oxidation Method in Order to Convert Tyrosol in Hydroxytyrosol. US Patent Application.

Violi, F., Loffredo, L., Pignatelli, P., Angelico, F., Bartimoccia, S., Nocella, C., Cangemi, R., Petruccioli, A., Monticolo, R., Pastori, D., and Carnevale, R. (2015). Extra virgin olive oil use is associated with improved postprandial blood glucose and LDL cholesterol in healthy subjects. Nutr. Diabetes 5: e172-e177.

Visioli, F., and Galli, C. (2001). The role of antioxidants in the mediterranean diet. Lipid 36(Suppl 1): 49-52.

Visioli, F., Galli, C., Bornet, F., Mattei, A., Patelli, R., Galli, G., and Caruso, D. (2000). Olive oil phenolics are dose-dependently absorbed in humans. FEBS Letters 468: 159-160.

Vissers, M.N., Zock, P.L., Roodenburg, A.J., Leenen, R., and Katan, M.B. (2002). Olive oil phenols are absorbed in humans. J. Nutr. 132: 409417.

Vitaglione, P., Savarese, M., Paduano, A., Scalfi, L., Fogliano, V., and Sacchi, R. (2015). Healthy virgin olive oil: a matter of bitterness. Crit. Rev. Food Sci. Nutr. 55: 1808-1818.

Vitali Čepo, D., Radić, K., Jurmanović, S., Jug, M., Grdić Rajković, M., Pedisić, S., Moslavac, T., and Albahari, P. (2018). Valorization of Olive Pomace-Based Nutraceuticals as Antioxidants in Chemical, Food, and Biological Models. Molecules 23(8): 2070.

Vogel, P., Machado, I.K., Garavaglia, J., Zani, V.T., De Souza, D., and Morelo Dal Bosco, S. (2015). Polyphenols benefits of olive leaf (Olea europaea L.) to human health. Nutr. Hosp. 31: 1427-1433.

Vulcano, I., Halabalaki, M., Skaltsounis, L., and Ganzera, M. (2015). Quantitative analysis of pungent and anti-inflammatory phenolic compounds in olive oil by capillary electrophoresis. Food Chem. 169: 381-386.

Wang, S., Moustaid-Moussa, N., Chen, L., Mo, H., Shastri, A., Su, R., Bapat, P., Kwun, I., and Shen, C.L. (2014). Novel insights of dietary polyphenols and obesity. J. Nutr. Biochem 25: 1-18.

Weinbrenner, T., Fito, M., Farre, A.M., Saez, G.T., Rijken, P., Tormos, C., Coolen, S., De la Torre, R., and Covas, M.I. (2003). Bioavailability of phenolic compounds from olive oil and oxidative/antioxidant status at postprandial state in healthy humans. Drugs under Experimental and Clinical Research 30: 207-212.

Wijayanthie, N., Gunarti, D.R., Manikam, N.R., and Yulhasri (2019). Efffects of extra virgin olive oil versus rice bran oil on glycemic control in patients with type -2 diabetes mellitus. Int. J. App. Pharm. 11(6): 56-59.

Wilhelm, J., Mehdi, B., and Christinae, R.-E. (2003). Process for the Isolation of Antioxidants. Patent EP1310175 A.

Yancey, P.G., Bortnick, A.E., Kellner-Weibel, G., de la Llera-Moya, M., Phillips, M.C., and Rothblat, G.H. (2003). Importance of different pathways of cellular cholesterol efflux. Arterioscler Thromb Vasc Biol. 23 712-719.

Yao, M., McClements, D.J., and Xiao, H. (2015). Improving oral bioavailability of nutraceuticals by engineered nanoparticle-based delivery systems. Current Opinion in Food Science 2: 14-19.

Yu, X., Zhu, P., Zhong, Q., Li, M., and Ma, H. (2014). Subcritical water extraction of antioxidant phenolic compounds from xiLan olive fruit dreg. Journal of Food Science and Technology 8: 5012-5020.

Zagklis, D.P., Vavouraki, A.I., Kornaros, M.E., and Paraskeva, C.A. (2015). Purification of olive mill wastewater phenols through membrane filtration and resin adsorption/desorption. J. Hazard. Mater. 285: 69-76.

Zhu, L., Liu, Z., Feng, Z., Hao, J., Shen, W., Li, X., Sun, L., Sharman, E., Wang, Y., Wertz, K., Weber, P., Shi, X., and Liu, J. (2010). Hydroxytyrosol protects against oxidative damage by simultaneous activation of mitochondrial biogenesis and phase II detoxifying enzyme systems in retinal pigment epithelial cells. The Journal of Nutritional Biochemistry 21: 1089-1098.

Zoidou, E., Melliou, E., Gikas, E., Tsarbopoulos, A., Magiatis, P., and Skaltsounis, A.L. (2010). Identification of throuba Thassos, a traditional Greek table olive variety, as a nutritional rich source of oleuropein. J. Agric. Food Chem. 58: 46-50. 